\title{
El estudio de las redes personales: contribuciones, métodos y perspectivas
}

\author{
JOSÉ LUIS MOLINA GONZALEZ \\ Universidad Autónoma de Barcelona \\ joseluis.molina@uab.es
}

Recepción: septiembre 2005

Aceptacion: noviembre 2005

\section{INTRODUCCIÓN}

¿Qué sentido tiene estudiar las redes personales más allá de mejorar las posibilidades de mercantilización de las relaciones sociales ${ }^{1}$ ? La respuesta que proponemos es la siguiente: a partir del estudio de las redes personales podemos avanzar en la comprensión de fenómenos sociales de rango «intermedio» o «meso» (Ferrand, 2002; de Federico, 2002), es decir, fenómenos en los que se presentan simultáneamente interacciones individuales, instituciones y estructuras sociales observables empíricamente. Efectivamente, en las redes personales podemos observar interacciones institucionalizadas (intercambios culturalmente dependientes de regalos entre familiares, de apoyo entre hijos adultos y sus padres, relaciones expresivas entre amigos ... pero también relaciones clienteproveedor, jefe-subordinado, político-elector) en el marco de estructuras sociales preexistentes (estratificadas por clase social y/o estatus, grupo ocupacional o residencial) que las influyen, condicionan o permiten. Al mismo tiempo, estas interacciones, ordenadas, unen simultáneamente redes de personas y redes de organizaciones (de todo tipo) en las que estas personas trabajan o participan, por lo que problemas tales como la acción colectiva, la movilización de recursos o la agenda política se hallan presentes en esta formulación ( $C f$. Requena Santos, 2003; Rodríguez, 1995; Scott, 1991; Wasserman et. al. 1994).

Esta conceptualización de la vida social inmediata no presupone ninguna teoría determinada de la acción social o económica en concreto (neoclásica o neoinstitucional) aunque evidentemente recoge la propuesta de Coleman (1990) y Bourdieu (1977) entre otros de las relaciones micro-macro o habitus-campo para poder dar cuenta de los fenómenos sociales. Bajo nuestro punto de vista, su ventaja reside en su empirismo y dualidad (Breiger, 1974), ya que podemos estudiar

${ }^{1}$ Esta cuestión es tratada con detalle en Petrizzo \& Maya, 2004. 
simultáneamente fenómenos micro (las interacciones) y fenómenos meso (las estructuras locales en las que éstas se hallan inmersas, incluidas las instituciones).

Diferentes fenómenos meso pueden ser estudiados desde esta perspectiva: las «comunidades», el «clientelismo» y los «nuevos movimientos sociales», por ejemplo. Naturalmente, nuestra perspectiva se añade a otras muchas posibles. La diferencia reside en tomar las interacciones sociales como punto de partida para identificar empíricamente estructuras en las que éstas se hayan insertas (Lozares, 2003). Estudiar las redes personales es pues, desde el punto de vista de la teoría social, relevante.

Además, las redes personales son especialmente importantes en el siglo XXI. En un momento en el que el capitalismo flexible (Castells, 1996) sigue avanzando en su mercantilización de todas las esferas de la vida social, incluidas por supuesto las relaciones personales, florecen las «comunidades» por doquier, las «étnicas», las «transnacionales», las «cibernéticas», las «académicas», las «instantáneas»... Por supuesto, la individualización de la vida social y la aparición de comunidades especializadas y parciales, son fenómenos relacionados. Barry Wellman (2001) ha llamado a esta forma de sociedad, después de décadas de estudio de la evolución de las relaciones personales, «Networked Individualism», o «Personalized Networking», algo así como «individualismo conectado». Es decir, el individuo y no el lugar, la familia o el grupo, ha pasado a ser el centro de las comunicaciones y de la vida social. Este individuo participa en una variedad de «comunidades» con las que tiene un compromiso variable y parcial, en modo alguno completo o absorbente como supuestamente ocurría en la «Comunidad» a la que hacía referencia Tönnies (1887) cuando la oponía a la «Asociación» urbana, o Robert Redfield (1960) con la «Pequeña Comunidad» campesina opuesta a la sociedad dominante, también urbana. Estas comunicaciones, ya sean cara a cara o mediadas por diferentes dispositivos (ordenadores, teléfonos -móviles o no) y programas (correo electrónico, chats, fórums, mensajería instantánea, blogs, SMS, etc.), se apoyan mutuamente, contribuyendo al mantenimiento y desarrollo de una red personal compuesta de relaciones soportadas por una variedad de medios (Wellman y Gulia, 1999).

Por último, el estudio de las redes personales nos puede permitir avanzar definitivamente en la comprensión de fenómenos tan relevantes hoy día como la identidad y los discursos étnicos (Molina y Aguilar, 2004; de Federico, 2004), las redes de apoyo de los inmigrantes (Maya Jariego et. al, 1999) y, por supuesto, todos los temas relacionados con la salud, el capital social y el desarrollo comunitario, entre otros.

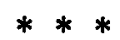

En este trabajo nos proponemos realizar un compendio, forzosamente limitado y provisional, de la investigación realizada en este campo. Para ello estudiaremos en primer lugar las diferentes tradiciones existentes dentro de la perspectiva egocéntrica (ver infra). Estas tradiciones empiezan en la Escuela de 
Manchester y llegan hasta una parte de los estudios centrados en el capital social, especialmente la impulsada por Nan Lin (2001). El segundo lugar intentaremos sistematizar los principales hallazgos en el campo de las redes personales atendiendo a seis dimensiones: tamaño, composición, tipos de relaciones, contenido de las relaciones, estructura y dinámica. En tercer lugar, recogeremos las principales conclusiones de la rica tradición de estudios centrados en analizar la fíabilidad y exactitud de las relaciones sociales recordadas, es decir, los sesgos sistemáticos que se producen cuando los informantes nos explican sus relaciones sociales. Dado que los informes de los egos sobre sus propias relaciones y sobre las relaciones de las personas de su red son la principal fuente de información utilizada, entendemos que esta información es de enorme interés. En cuarto lugar, presentaremos la propuesta de Chris McCarty (2002) para recoger y analizar la estructura de la redes personales con la ayuda del programa Egonet ${ }^{2}$. Entendemos que esta propuesta nos permite avanzar en el estudio de las redes personales de una forma nueva y penetrante, al tiempo que hace viable la recolección de datos de otra forma muy difíciles de obtener. Por último, reservamos el quinto apartado para reflexionar sobre las alternativas disponibles para la integración de los niveles micro y meso en el análisis de la realidad social.

\section{LAS TRADICIONES SOCIOCÉNTRICA Y EGOCÉNTRICA}

De la literatura de análisis de redes sociales (ARS, $C f$. Ilustración 1) pueden derivarse dos aproximaciones distintas: la sociocéntrica y la egocéntrica (McCarty 2002a). Estas perspectivas pueden remontarse hasta Simmel (1922) y Moreno (1934) para la primera y posiblemente Bott (1955) y Epstein (1961) para la segunda ${ }^{3}$. La aproximación sociocéntrica es bien conocida: explica las propiedades de un grupo de conexiones existentes entre un grupo de nodos definidos previamente tanto por un criterio realista (por la existencia de una entidad social preexistente, un equipo de fútbol, una clase, una organización) como por un criterio nominalista (introducido por el investigador, Laumann, 1983). La aproximación egocéntrica, en cambio, parte de las conexiones que se pueden trazar a partir de un ego dado. En el caso de Bott, el punto de anclaje (el «ego», en este caso) eran las unidades domésticas de parejas inglesas; en el caso de Epstein era un individuo, un informante africano varón saludando personas pertenecientes a diferentes grupos étnicos en una ciudad de la antigua Rodhesia. Con algunas excepciones (Molina et. al, 2001) este último sentido es el normalmente utilizado en las «redes egocéntricas», expresión actualmente intercambiable con la de «redes personales» (Lonkila 1998).

${ }^{2}$ http://survey.bebr.ufl.edu/EgoNet/index.shtml

${ }^{3}$ Radcliffe Brown (1940) y Barnes (1954) quienes inventaron el término «red social» , estaban más interesados en la estructura global de la sociedad o una colectividad que en las características de las redes personales. 


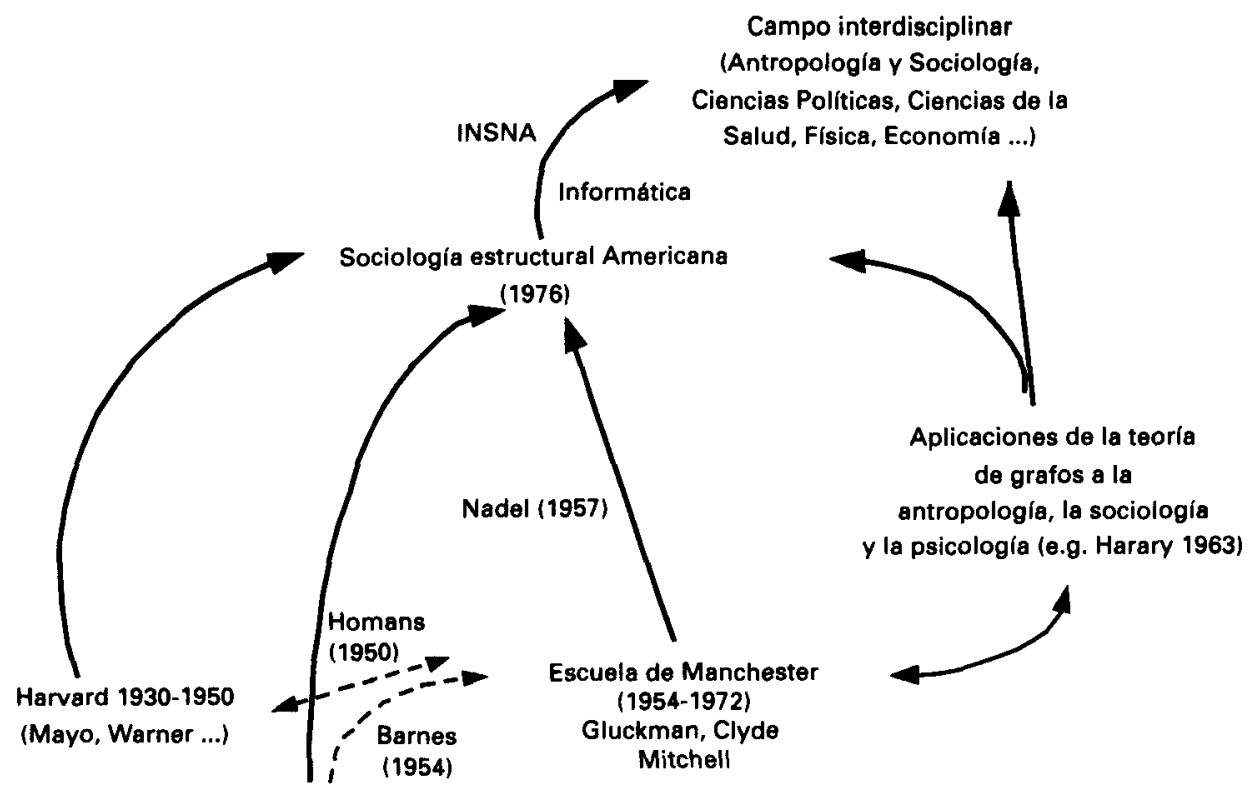

Sociometría de Moreno (1934)

Ilustración 1. El desarrollo del análisis de redes sociales (Cf. Freeman, 2004).

\section{LA APROXIMACIÓN EGOCÉNTRICA}

En este apartado intentamos identificar las principales tradiciones de investigación en el análisis de las redes personales y sintetizar sus principales contribuciones. Por supuesto, asignar etiquetas es siempre una tarea problemática, pero pensamos que pueden ayudarnos a disponer de la perspectiva global que buscamos.

La Tabla 1 muestra las principales características de las cuatro tradiciones identificadas: la Escuela de Manchester, los Estudios de Comunidad, lo que he llamado «Estudios de estimación del tamaño de las redes personales» y una parte importante de los estudios de Capital Social. Por supuesto, la lista de trabajos no intenta ser un catálogo exhaustivo sino tan sólo una guía de las principales contribuciones.

\section{La Escuela de Manchester}

Los antropólogos urbanos de la Escuela de Manchester estaban interesados en las redes sociales para explicar el comportamiento que no podía ser explicado desde un paradigma teórico estructural-funcionalista, el cual postulaba la existencia de una red activa de instituciones que permitía dar cuenta de la vida 
Tabla 1

Las cuatro tradiciones de la aproximación egocéntrica

\begin{tabular}{|c|c|c|c|}
\hline Tradición & Enfoque teórico & Principales estudios & Métodos \\
\hline $\begin{array}{l}\text { ESCUELA DE } \\
\text { MANCHESTER }\end{array}$ & $\begin{array}{l}\text { Complemento del } \\
\text { paradigma del } \\
\text { estructural } \\
\text { funcionalismo en un } \\
\text { mundo urbano } \\
\text { fluido. }\end{array}$ & $\begin{array}{l}\text { Barnes }(1954) \\
\text { Bott }(1955,1957) \\
\text { Epstein }(1957,1963) \\
\text { C. Mitchell }(1969) \\
\text { Boissevain }(1973) \\
\text { Kapferer }(1972)\end{array}$ & $\begin{array}{l}\text { Sociogramas, } \\
\text { observación } \\
\text { participante, } \\
\text { conceptos sobre } \\
\text { teoría de grafos y } \\
\text { álgebra de matrices. }\end{array}$ \\
\hline $\begin{array}{l}\text { ESTUDIOS DE } \\
\text { COMUNIDAD }\end{array}$ & $\begin{array}{l}\text { Lazos comunitarios } \\
\text { más allá de los } \\
\text { límites residenciales, } \\
\text { apoyo social y } \\
\text { cambio de la red } \\
\text { personal a lo largo } \\
\text { del tiempo. }\end{array}$ & $\begin{array}{l}\text { Laumann (1973) } \\
\text { Fisher }(1982) \\
\text { Wellman }(1979, \\
\text { 1982, 1988, 1997, } \\
\text { 1999) } \\
\text { Litwin (1996) } \\
\text { Tilburg (1998) } \\
\text { Ferrand (1999) }\end{array}$ & $\begin{array}{l}\text { Grandes encuestas } \\
\text { egocéntricas. Bases } \\
\text { de datos públicos } \\
\text { con datos de redes } \\
\text { sociales. }\end{array}$ \\
\hline $\begin{array}{l}\text { ESTIMACIÓN DEL } \\
\text { TAMAÑO DE LAS } \\
\text { REDES PERSONALES }\end{array}$ & $\begin{array}{l}\text { Tamaño, estructura o } \\
\text { muestras } \\
\text { representativas de } \\
\text { redes personales. }\end{array}$ & $\begin{array}{l}\text { Poole y Kochen } \\
\text { (1978) } \\
\text { Killworth y Bernard } \\
(1978,1984) \\
\text { Killworth et. al. } \\
(1998,1990) \\
\text { Freeman y } \\
\text { Thompson }(1989) \\
\text { Bernard }(1990,1998) \\
\text { McCarty }(1997,2000)\end{array}$ & $\begin{array}{l}\text { Muestras de guías } \\
\text { telefónicas locales o } \\
\text { listas de nombres, } \\
\text { "Mundo Pequeño al } \\
\text { revés, RSW», } \\
\text { método «Scale-up». }\end{array}$ \\
\hline CAPITAL SOCIAL & $\begin{array}{l}\text { Acceso a personas } \\
\text { en posiciones } \\
\text { sociales superiores y } \\
\text { sus recursos } \\
\text { asociados. }\end{array}$ & $\begin{array}{l}\text { Lin }(1982,2001) \text {, } \\
\text { Lin et. al. (2001), } \\
\text { Burt (1992), Flap et. } \\
\text { al. (1999), van der } \\
\text { Gaag y Snijders } \\
\text { (2003) }\end{array}$ & $\begin{array}{l}\text { Generador de } \\
\text { nombres a partir de } \\
\text { posiciones sociales, } \\
\text { generador de } \\
\text { nombres a partir de } \\
\text { recursos accesibles. }\end{array}$ \\
\hline
\end{tabular}

social. Estos antropólogos insistieron en la idea de que sus estudios eran un complemento a las debilidades de la teoría en emplazamientos urbanos donde podía encontrarse una «confusion» de lenguajes, instituciones y grupos étnicos. La perspectiva egocéntrica fue ampliamente adoptada como herramienta de investigación con la notable excepción de Barnes, más interesado en una perspectiva sociocéntrica y de clase social. Aparte de la famosa correlación de Bott entre el grado de segregación de los roles conyugales y la densidad de las redes sociales de las parejas londinenses estudiadas, estos antropólogos urbanos desarrollaron interesantes indicadores de redes personales ( $C f$. Mitchell 1969) y acordaron la existencia generalizada de un núcleo íntimo y efectivo (con un alta densidad de relaciones) y un círculo extendido de conocidos menos denso (ver el ejemplo de 
la Ilustración 2). Como veremos más adelante, esta visión de las redes personales no solamente ha sido ampliamente respaldada empíricamente (Suitor et. al, 1997; Morgan et. al., 1997) sino que refleja la visión sesgada que los informantes tienen de sus propias redes (Kumbasar et al. 1994:499) con ego en el centro de un núcleo denso de relaciones y una periferia dispersa de conocidos. Otra tradición, la de Estudios de Comunidad, también parte de esta visión de las redes personales (ver la conocida imagen realizada por Barry Wellman de la red social de un habitante de East York en la Ilustración 3).

Estos investigadores documentaron la relación entre la estructura de la red

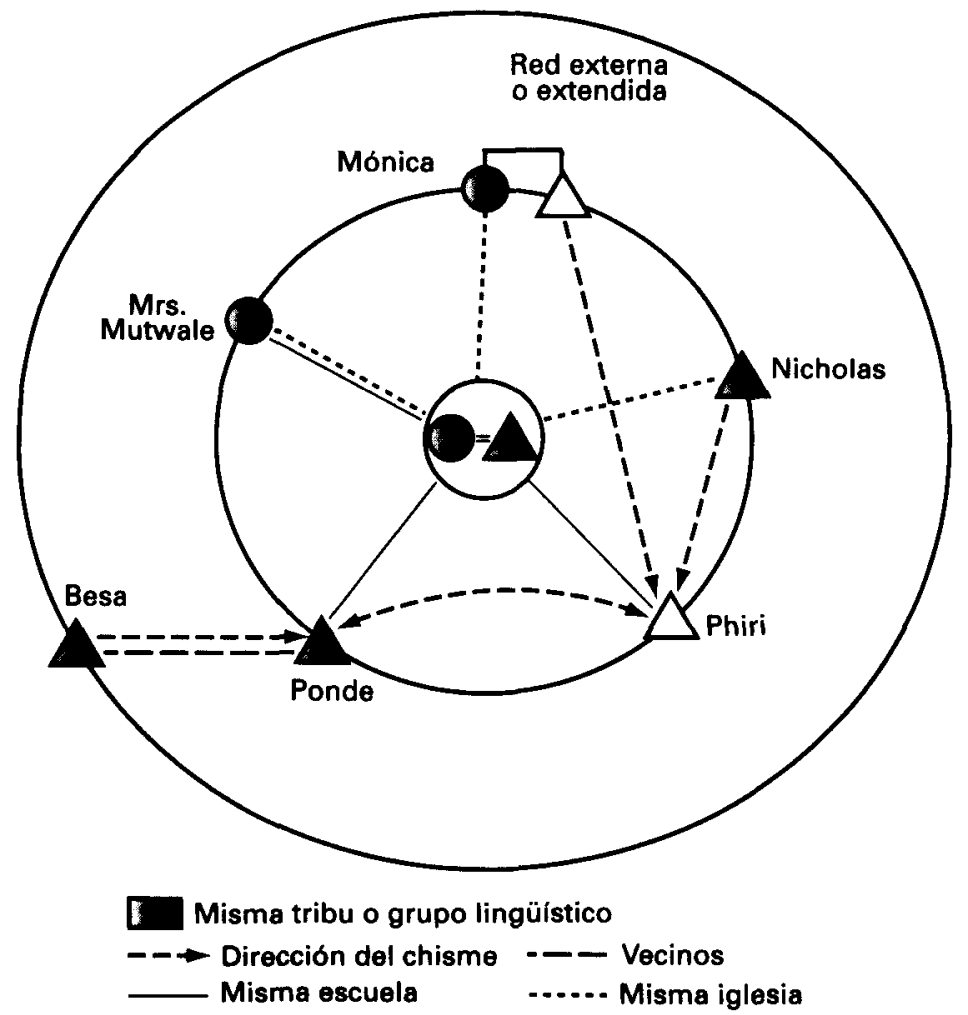

Ilustración 2. La red de habladurias. Adaptado de Epstein, 1969.

personal y la conducta individual en situaciones basadas en clientelismo, en luchas políticas y en conflictos sociales en lugares de trabajo. Además, observaron las diferencias de género en la extensión y estructura de redes personales, básicamente por razones ocupacionales: los hombres tenían redes mayores y menos densas porque sus trabajos proporcionaban un amplio rango de nuevos contactos (Kapferer, 1973). 


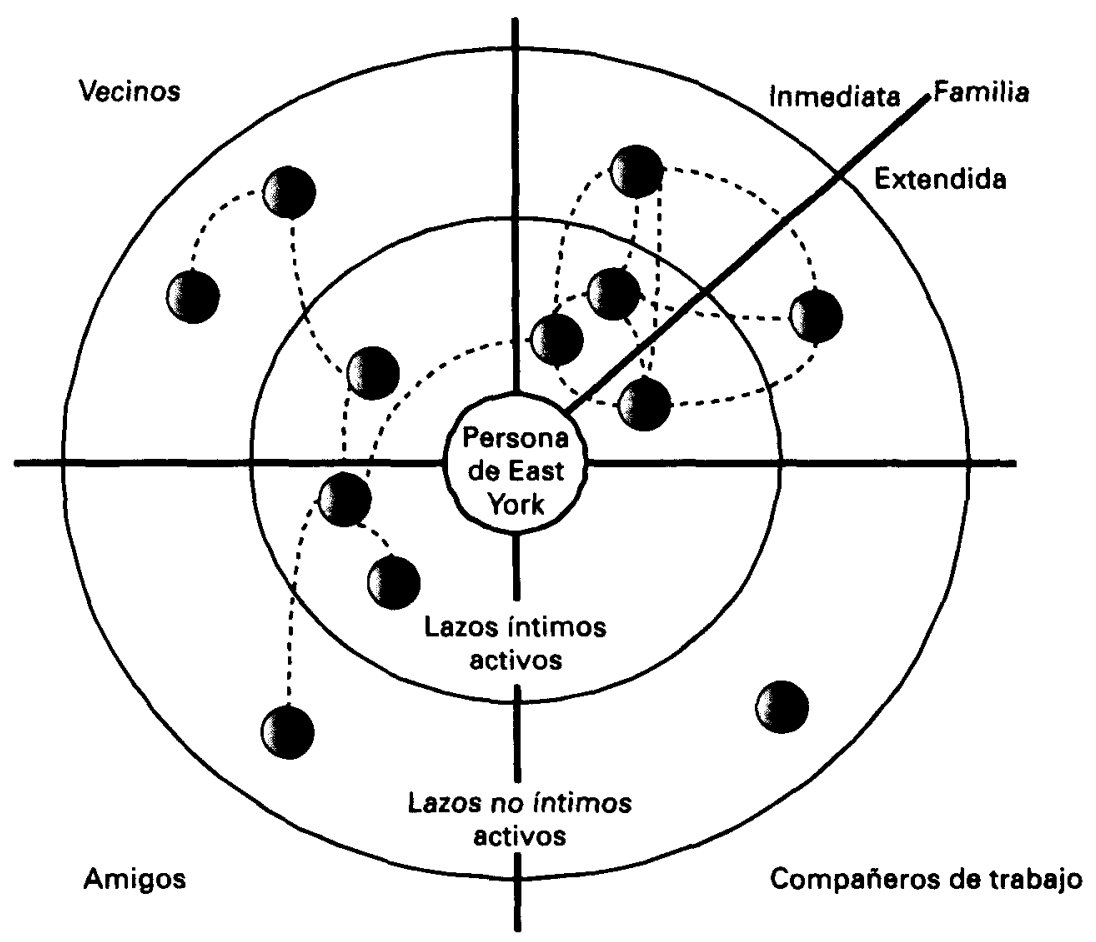

Ilustración 3. Red personal típica de una persona de East York. Adaptado de Wellman, 1999.

La Escuela de Manchester influyó en el trabajo de Nadel, Theory of Social Structure (1957), una formulación de la sociedad como una red de roles, el cual influyó a su vez en el pionero trabajo de Harrison White (1976) sobre equivalencia estructural ( $C f$. Ilustración 1). Sin embargo, la falta de capacidad de cómputo informático hizo imposible el desarrollo de este campo de investigación. El técnicamente sofisticado trabajo de Bruce Kapferer en una fábrica en Zambia (1972), con análisis de matrices y índices de redes de poder e influencia, fue el último logro de esta tradición.

Markku Lonkila $(1997,1998)$ ha desarrollado más recientemente un interesante trabajo basado en la comparación de dos grupos informantes (Rusia y Finlandia) usando principalmente agendas de contactos. Lonkila pidio a 40 profesores de secundaria de San Petersburgo y 38 de Helsinki que anotasen diariamente durante dos semanas en un cuestionario estructurado las relaciones significativas que habían tenido durante el día. Una vez acabado el período se les pidió que añadiesen las personas que no figuraban en la lista pero que creían importantes. Además, se realizaron entrevistas en profundidad para documentar la historia de vida de cada informante. Esta investigación muestra la cambiante na- 
turaleza de las redes personales en un contexto post-comunista y las diferentes estrategias utilizadas para adaptarse a las nuevas circunstancias (ya sea fortaleciendo los vínculos propios de la era comunista o ampliando nuevos contactos en una sociedad más orientada al mercado). Aunque singular, este trabajo parece compartir el interés de los antropólogos de la Escuela de Manchester por captar el papel de las redes personales en una sociedad en rápida transformación.

\section{Los Estudios de Comunidad}

Los cambios en la forma de vida tradicional no solamente preocuparon a los antropólogos urbanos en África del Sur y otros lugares del mundo, sino que también sociólogos americanos en los setenta y ochenta se ocuparon de la llamada «Cuestión Comunitaria» (Cf. Wellman, 1979), la transformación de la comunidad en la sociedad «moderna» y urbana. Esta tradición de investigación se centra en la localización de las redes de apoyo social, redes de iguales constituidas por parientes, amigos y vecinos que proporcionan socialización, información y ayuda en general. Laumann (1973) Fisher (1982), el Proyecto East York iniciado por Barry Leighton y Barry Wellman en 1968 y que continuo hasta los noventa, Schweizer et. al (1998) en California, proporcionaron una gran base empírica para formular las características globales de redes personales en la sociedad americana y canadiense. Los límites residenciales eran todavía importantes, pero estaban constantemente atravesados por cartas, llamadas de teléfono y redes de transporte. Más recientemente, internet proporcionó más oportunidades para complementar las interacciones cara a cara y mantener activas las relaciones, relaciones que, gracias a los estudios longitudinales, sabemos que cambian durante el tiempo. Tendremos oportunidad de sistematizar estos hallazgos más adelante. Ahora nos interesa resaltar que el método utilizado por esta aproximación fue la encuesta, complementada con entrevistas en profundidad con informantes clave. La lista de preguntas usadas por los investigadores para obtener las redes sociales de las personas entrevistadas, los generadores de nombres, tendían lógicamente a identificar las redes de apoyo o los lazos fuertes en lugar de los débiles.

Cabe mencionar que este énfasis en el cambio y el uso de grandes encuestas ha sido aplicado también en el estudio del apoyo social en las redes de las personas mayores (Tilburg 1992, 1998; Litwin 1996, Ferrand 1999). Por término medio, la red social se incrementa continuamente hasta la madurez para decrecer hasta los sesenta o más tarde, con una creciente importancia de los parientes y los lazos íntimos (que sustituyen a los compañeros de trabajo y los vecinos) y de los roles de ayuda instrumental y emocional. Sin embargo Litwin (1996:220) calculo en su resumen de un estudio comparativo trasnacional que un tercio de las personas mayores no tenía ayuda informal en absoluto y que, paradójicamente, esas personas no tenían acceso a los servicios sociales (posiblemente por falta de información).

EMPIRIA. Revista de Metodología de Ciencias Sociales. N..$^{\circ}$ 10, julio-diciembre, 2005, pp. 71-105. 
Veamos un ejemplo de la metodología utilizada en estos estudios $(C f$. Requena, 1996) a partir de la encuesta utilizada por Fisher en 1977 en California, posiblemente el más completo por lo que se refiere a los generadores de nombres utilizados ${ }^{4}$ :

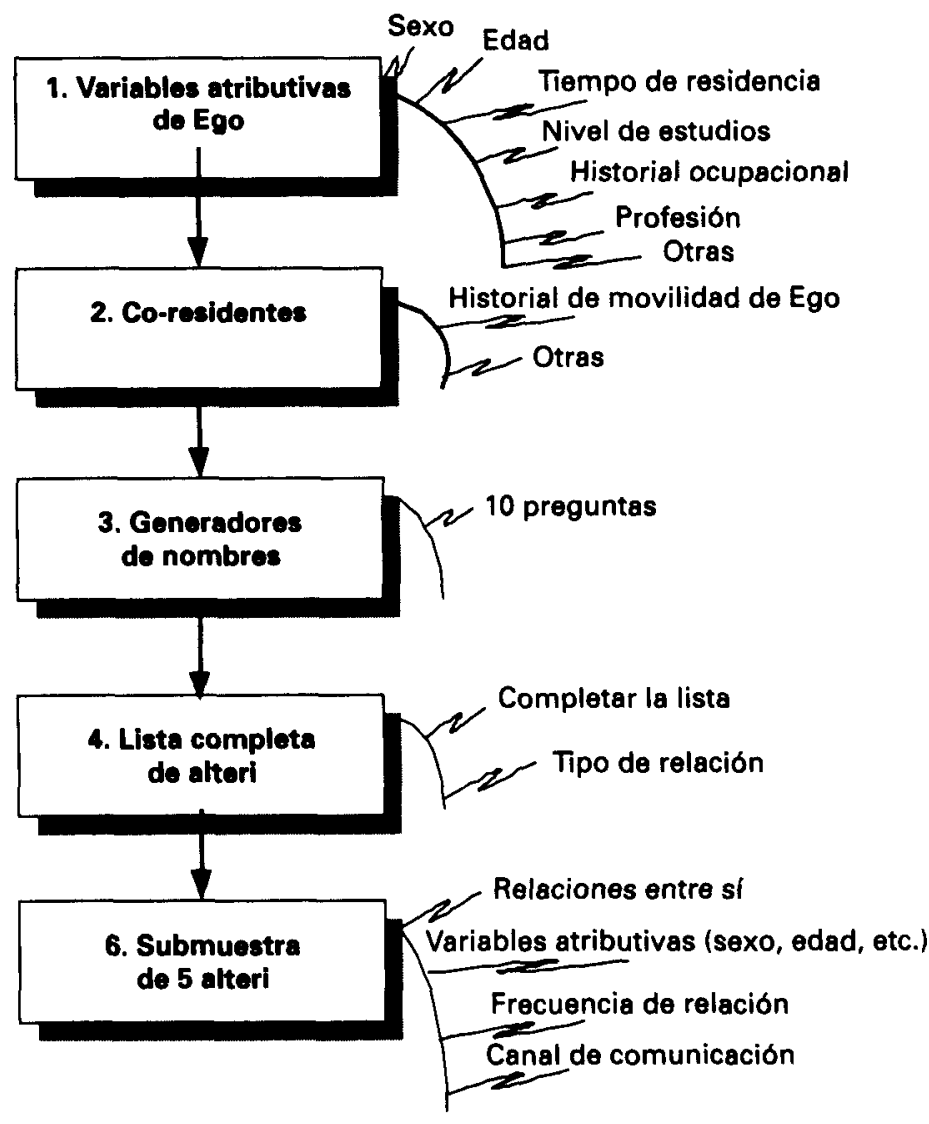

Ilustración 4. Metodología de la encuesta de redes personales de Fisher (Cf. Grossetti, 2004).

Este tipo de encuestas exige alrededor de una hora de tiempo para completarse. Como puede observarse se recogen en primer lugar variables atributivas de ego y, en este caso, información sobre las personas que residen en su misma casa. Los generadores de nombres utilizados pretenden recoger relaciones de una

${ }^{4}$ Agradezco a Michel Grossetti su amabilidad en proporcionarme la encuesta utilizada en la investigación realizada en Toulouse, una réplica de la realizada por Fisher en San Francisco, de gran interés comparativo (Grossetti, 2004).

EMPIRIA. Revista de Metodología de Ciencias Sociales. N. ${ }^{\circ}$ 10, julio-diciembre, 2005, pp. 71-105. 
variedad de escenarios sociales (domésticos, profesionales, ocio, ayuda de emergencia, etc.). De forma resumida y aproximada éstos son los siguientes (se realiza siempre una pregunta previa para evitar que la respuesta sea »nadie» y poder pasar a la siguiente):

1. Si tuviese que ausentarse temporalmente de la ciudad, ¿a quién le pediría que se ocupase de regar las plantas, recoger el correo o simplemente de echar un vistazo?

2. ¿Con quién habla de temas relacionados con el trabajo?

3. En los últimos tres meses, ¿a quién ha ayudado en tareas domésticas como cocinar, mover muebles, lavar o realizar pequeñas reparaciones?

4. ¿Con quién ha compartido alguna de las siguientes actividades en los últimos tres meses (comer o cenar en casa, comer o cenar fuera, recibir visitas, visitar, encontrarse en algún lugar fuera de casa como un bar o restaurante ...)?

5. ¿Con quién discute regularmente de temas relacionados con el ocio o aficiones en común?

6. ¿Nos puede decir las iniciales de su novio/a o de su mejor amigo/a con la que se vea muy a menudo? (en el caso de que se trate de una persona casada se piden los años de convivencia)

7. Cuando comparte sus problemas personales, ¿con quién lo hace?

8. Si tuviese que tomar una decisión importante referente a la familia o el trabajo ¿Con quién la discutiría?

9. Si tuviese que pedir prestada una importante cantidad de dinero a un conocido ¿a quién lo haría?

10. ¿Hay personas importantes para Ud. que haya tenido algún contacto en los dos últimos años y que no aparezcan en la lista?

Los nueve primeros generadores de nombres produjeron en el estudio de California una media de 12,8 nombres por respondiente y el generador 10 una media de 5,7 alteri (personas nominadas) adicionales. La media total en el estudio de Fisher fue de 18,5 personas (Grossetti, 2004). De cada una de las personas nombradas se pide el tipo de relación existente (amistad, familiar, conocidos, etc.).

A continuación, de esa lista se selecciona una submuestra de 5 personas escogiendo el primer nombre aparecido en 5 de los 10 generadores, se investiga si tienen relaciones entre sí (para hallar la densidad) y se preguntan nuevas variables atributivas de esas personas (parecidas a las de ego) además de la frecuencia de interacción con ego y el canal de comunicación (cara-a-cara, teléfono y, en la encuesta de Toulouse, correo electrónico).

Como puede verse estas encuestas son muy costosas de realizar y, aunque permiten disponer de una visión rigurosa y representativa de la composición de las redes personales, los datos disponibles sobre la estructura de relaciones se reducen a una submuestra mínima. 
Con algunas variaciones, pero siguiendo en general la propuesta de indagar sobre la ayuda cotidiana, la ayuda de emergencia, el apoyo emocional, las relaciones derivadas del trabajo, del ocio y pedir el tipo, la frecuencia y el canal de comunicación, se han desarrollado encuestas periódicas en diferentes estados. Cabe destacar por su valor comparativo la encuesta coordinada por el ISSP (International Social Survey Programme <www.issp.org>) sobre redes sociales y sistemas de apoyo en 1986, con la participación de las agencias de Australia, Austria, Reino Unido, Hungría, Italia, Estados Unidos y Alemania Occidental. El ISSP coordina grandes encuestas periódicas estatales de forma que los datos sean comparables. La última encuesta comparativa sobre redes personales a nivel internacional se realizó en el 2001 y los datos disponibles abarcan 34 estados. En general, la encuesta de 2001 sigue el esquema de la 1986, con algunos nuevos generadores de nombres.

Tabla 2

Encuestas sobre redes personales ISSP

\begin{tabular}{|c|l|l|}
\hline Preguntas & \multicolumn{1}{|c|}{ Contenido } & \multicolumn{1}{c|}{ Comentario } \\
\hline $1-13$ & Contacto con la familia inmediata & ISSP-1986 e ISSP-2001 \\
\hline 14 & $\begin{array}{l}\text { Contacto con otros familiares (incluidos } \\
\text { padrinos-madrinas) }\end{array}$ & ISSP-2001 \\
\hline $15-17$ & $\begin{array}{l}\text { Buenos amigos en el trabajo, en el barrio, } \\
\text { otros amigos }\end{array}$ & $\begin{array}{l}\text { ISSP-2001 (en la encuesta } \\
\text { ISSP-1986, número total } \\
\text { de buenos amigos) }\end{array}$ \\
\hline $18-20$ & Contacto con los mejores amigos & ISSP-1986 e ISSP-2001 \\
\hline 21 & Participación en asociaciones o grupos & ISSP-2001 \\
\hline $22-28$ & Ayuda en caso de necesidad & ISSP-1986 e ISSP-2001 \\
\hline 29 & Información sobre oportunidades de trabajo & ISSP-2001 \\
\hline $30-39$ & $\begin{array}{l}\text { Cuestiones varias sobre satisfacción, } \\
\text { confianza, opiniones políticas, etc. }\end{array}$ & ISSP-2001 \\
\hline
\end{tabular}

Evidentemente, estas informaciones se completan con un amplio conjunto de variables atributivas de los respondentes. La información disponible es de enorme interés al poder cruzar esas variables con la composición de las redes personales y el tipo de contenido de las relaciones existentes. Sin embargo, estas encuestas no incluyen la información de quién conoce a quién de entre los alte$r i$, es decir, no permiten conocer la estructura de relaciones de la red personal, sino solamente aproximaciones de las «zonas» primaria y extendida. Para disponer de esta información es necesario adoptar otra estrategia que veremos más adelante. 
En conjunto pues, nos encontramos con una tradición de estudios bien consolidada en la teoría, los métodos y con amplias bases de datos comparativas disponibles que nos permiten conocer las características globales de las redes personales en prácticamente todo el mundo. Sin duda, una gran contribución. Pasemos ahora a la siguiente tradición de estudios.

\section{Estudios de estimación del tamaño de las redes personales}

¿A cuántas personas conoces? La respuesta a esta sencilla pregunta ha dado lugar a una tradición de estudios centrada en la estimación del tamaño de las redes personales. Ithiel de Sola Pool y Manfred Kochen en un borrador de su artículo "Contactos e influencia» (1978) presentaron una serie de experimentos para investigar esta cuestión y propusieron una agenda de investigación. Otro de los resultados más conocidos de esta línea de trabajo fueron los experimentos de Mundo Pequeño llevados a cabo por Milgram (1967) y toda la literatura derivada desde entonces (Watts, 1999; Barabási, 2002; Newman, 2003).

El primer experimento consistió en un cálculo del volumen total de conocidos de una persona utilizando dos guías telefónicas locales, una de Manhattan y otra de Chicago. El sujeto fue el mismo Pool. De cada guía se seleccionaron treinta páginas y se buscó personas conocidas en ellas. Las estimaciones arrojaron un total de 3.100 personas utilizando la guía de Chicago y 4.250 de la de Manhattan. Esta discrepancia fue explicada en parte porque Pool era un judío que creció en Manhattan donde esta población está más representada que en Chicago. Este cálculo está claramente basado simplemente en «conocer» y no en interacciones efectivas o en la existencia de un reconocimiento mutuo.

Insatisfecho con esta aproximación Pool inició un diario de contactos interpersonales. Después de 100 días de registrar las interacciones, los datos fueron tabulados con la proporción de nuevos nombres que aparecían en la lista a lo largo del tiempo. La curva en los últimos días fue prácticamente cero. Extrapolando la cantidad de conocidos a lo largo de un período de 20 años, la cifra resultante fue de 3.500. A pesar que esta cifra está basada en interacciones efectivas, muchas relaciones desaparecen con el tiempo como los autores señalaron (un experimento mostró que, después de 2 años, un $25 \%$ de los contactos previamente registrados se habían olvidado).

Esta técnica fue aplicada por un alumno de Pool, Michael Gurevich (1961) para recopilar datos en 18 personas, cada una de las cuales llevaba un diario de contactos. La estimación variaba de 122 a 5.053 con una media de 2.130.

Otra aplicación de esta técnica fue la estimación de conocidos del Presidente Roosvelt a partir de su agenda de citas realizada por otro estudiante del MIT, Howard Rosenthal, que le llevó a estimar 22.500 contactos en 20 años ( $C f$. Freeman y Thompson, 1998).

La segunda serie de experimentos fueron llamados El Reverso del Mundo Pequeño (RSW) (Killworth 1978, 1984; Bernard 1988). El RSW está inspirado 
por supuesto en el experimento del Mundo Pequeño de Milgram. Se mostraba a los informantes una lista de 500 nombres «míticos» o comunes (100 de ellos podían variar en cada experimento en función de la zona) junto con la ocupación y lugar de residencia de cada uno de ellos. A continuación intentaban establecer un camino probable hasta el contacto objetivo a partir de sus contactos personales. Gracias a la cantidad de conocidos obtenidos con este generador de nombres se podía calcular el tamaño de la red personal. La media resultante fue de 250. Al parecer esta técnica tendía a capturar sólo una parte de la red social (Freeman y Thompson, 1989).

Siguiendo esta línea de trabajo, estos investigadores adoptaron finalmente (Killworth et. al, 1998, 1990; McCarty et. al, 2000) una tercera estrategia para el cálculo del tamaño de las redes personales: el scale-up method, o el método del escalamiento proporcional a partir del número de personas conocidas por un respondiente de una subpoblación de tamaño conocido (e.g. diabéticos, indios americanos, muertos en accidente de tráfico). Este método asume que en condiciones normales la probabilidad que un alter de una subpoblación determinada figure en la red de ego es igual al tamaño de esa subpoblación en la población global, es decir, que si un $1 \%$ de una población tiene una característica determinada es de esperar que, en promedio, un $1 \%$ de las personas presentes en las redes personales presenten esta característica también. A partir de un listado de preguntas sobre personas conocidas en una amplia serie de subpoblaciones es posible obtener un estimador para cada informante.

Este método ha sido aplicado en múltiples encuestas de forma sistemática (test/re-test) por los mismos autores, utilizando diferentes subpoblaciones y nombres de pila (cuya frecuencia también es conocida) y contrastada con otro método de estimación del tamaño de la red personal como sumar las personas presentes en 16 tipos diferentes de relación (familia nuclear, otros familiares, compañeros de trabajo, amigos íntimos, buenos amigos, vecinos... y «otros»). Los resultados de las encuestas son sorprendentemente estables: la media de la red personal es de aproximadamente 291 contactos activos, con una desviación típica de 250. El generador de nombres utilizado en estas encuestas normalmente define «conocer» a una persona si ésta reconoce a ego por su nombre o por su aspecto, que ego puede contactar con él y que se han visto en alguna ocasión en los dos últimos años (Cf. McCarty et. al. 1997).

Esta estimación han sido usada recientemente para calcular el «efecto onda», es decir, la cantidad de personas que conocen a afectados por una tragedia o evento (Bernard et. al. 2001; Newman, 2001).

Por tanto, si sumamos lo que sabemos de las diferentes tradiciones podemos proponer que la media de los contactos activos de las redes personales, al menos en Estados Unidos (en México, por ejemplo, el número de contactos obtenidos fue menor, $C f$. Bernard et. al., 1990) es de unos 290, con una importante desviación típica debida seguramente a la estratificación social (las posiciones sociales más altas suelen poseer más y «mejores» contactos»), siendo también importante el sexo y la profesión. Esta definición de red personal es mucho más restrictiva 
que la de los contactos mantenidos a lo largo de la vida, es decir, las personas con las que se ha tenido relación en algún momento pero que no hay garantía de reconocimiento mutuo. Esta segunda definicion, la obtenida a partir de la proyección de agendas de contactos o de guías telefónica, arroja unas estimaciones de entre 1.500 y 3.500 personas (e incluso 4.707 en la estimación más extrema). Sin embargo, aún así la estimación derivada de la definición de los contactos activos es inmensamente mayor que la mayor de las estimaciones $(18,5)$ de las redes personales investigados por la tradición de los Estudios de Comunidad.

Cabe recordar que los generadores de nombres utilizados en esta última tradición tienden a recoger los lazos fuertes. Así, en la Ilustración 5 se presentan las diferencias entre las diferentes estimaciones, aunque las estimaciones de los contactos «conocidos» varían notablemente $(3.500,4.707$ y 22.500 para el presidente Roosevelt).

La más reciente aproximación al estudio del tamaño de las redes personales ha sido realizada por Fu (2005). En su estudio muestra cómo los resultados de las encuestas poblaciones en China, Taiwan y Hong-Kong que durante una década han incluido la pregunta ¿aproximadamente, con cuántas personas tiene contacto diariamente ? $^{5}$ son extremadamente estables, en torno a las 3,4 personas de media, con una desviación típica de 1,4. Por su parte, tres informantes lleva-

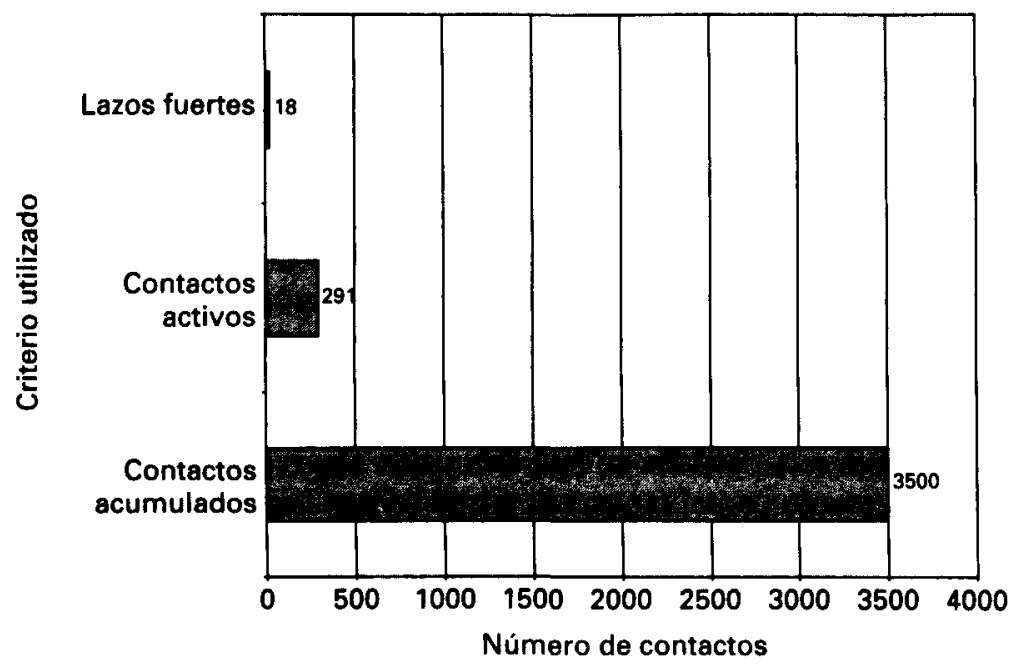

Ilustración 5. Estimaciones aproximadas de las redes personales según los criterios utilizados.

${ }^{5}$ On an average, about how many people do you have contact with in a typical day,including all those who you say hello, chat, talk or discuss matters with, whether you do it face-to-face, by telephone, by mail or on the internet and whether you personally know the person or not? Please give your estimate and select one from the following categories that best matches your estimate: (1) 0-4 persons, (2) 5-9 persons, (3) 10-19 persons, (4) $20-49$ persons, (5) 50-99 persons, (6) over 100 persons. 
ron detallados diarios de contactos durante 3-4 meses que dieron una media de 29 contactos diarios con una desviación de 9 personas.

La conclusión es evidente. No existe una única respuesta a la pregunta inicial de ¿cuántas personas conoces? Sin embargo, por diferentes medios podemos aproximarnos al conocimiento de las redes personales, su evolución y sus efectos en la vida social.

\section{Capital social y redes personales}

Sin pretender realizar una revisión exhaustiva de la literatura sobre capital social, sí podemos apuntar que ésta se puede dividir en tres grandes grupos: el capital social centrado en la persona, el capital social centrado en la red y el capital social centrado en la red de asociaciones cívicas. El primer uso (Bourdieu, 1977; Coleman, 1988) concibe el capital social como algo inherente a las personas, esto es, al número y la calidad de las relaciones de ego, determinados por su clase social. El segundo sentido del término de capital social hace residir los recursos en las propiedades de la red de relaciones, más que en las personas. Ronald Burt (1992) nos habla de la existencia de «agujeros estructurales» es decir, contactos no redundantes que confieren más poder $\mathrm{e}$ influencia a los nodos necesarios para establecer conexiones en la red. De esta forma, la estructura de la red social es la que determina el capital social. Cuanto mayor sea el grado de intermediación, es decir, la capacidad de ser «puentes» en la red, de conectar grupos de forma exclusiva, mayor será el capital social.

Finalmente, el tercer uso del concepto de capital social se debe a Putnam (1993, 1995), el cual asocia el éxito económico de una región de un país con la red de entidades cívicas y económicas existentes. Dicho de otra forma: la existencia de una densa red de organizaciones contribuye al éxito económico de una región. De esta forma explica el fenómeno de los distritos industriales en Italia y su desarrollo en contraste con las regiones del sur, a pesar de formar parte del mismo entorno económico y político.

La propuesta de Nan Lin se sitúa desde sus inicios (1982) en un término intermedio entre la primera y la segunda. Lin define el capital social como el conjunto de recursos inmersos en la red social (2001). Por tanto, estos recursos son tanto una propiedad de las redes personales (determinadas especialmente por su posición en la jerarquía social, o clase social) como por las características de las redes más amplias en las que se hayan inmersas, esto es, que aquéllas personas que ocupan posiciones de intermediación disponen efectivamente de más y mejores recursos. Este capital social dispone de dos momentos, el acceso, la capacidad social de acceder a los recursos y el uso, la captación efectiva de recursos realizada de forma intencional. Esta definición de capital social de Lin implica una concepción meso de análisis.

Para poder medir de forma adecuada el capital social definido de esta forma, es necesario conocer las posiciones sociales de los alteri por una parte y el nivel 
de acceso existente por la otra (es decir, si el acceso a esos recursos es completo, parcial o inexistente al ser una relación débil). Para ello Lin propuso medir esta capital mediante un generador de posiciones sociales en lugar de de mediante los generadores de nombres comúnmente utilizados. Efectivamente, las recursos sociales se hayan desigualmente distribuidos a partir de las posiciones sociales, dispuestas en forma piramidal.

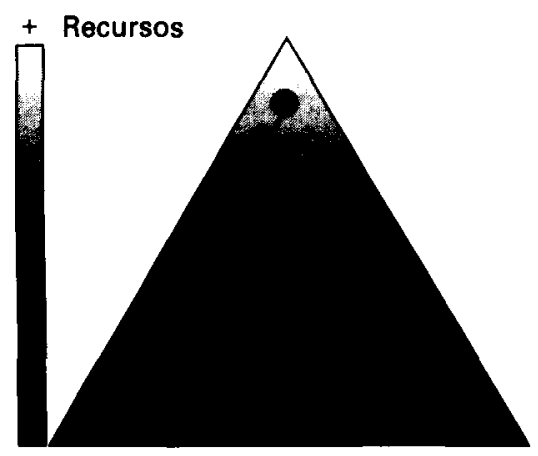

Ilustración 6. Distribución desigual de recursos en la estructura social.

Por tanto, a partir de una muestra conveniente de las profesiones y posiciones existentes en una estructura social dada y preguntando el número de personas conocidas en cada una de las posiciones, es posible obtener una aproximación al capital social potencialmente accesible. Si, además, se dispone de información sobre la intensidad de la relación con la persona que ocupa esa posición, se puede predecir el capital social movilizable en un momento dado por una acción intencional de ego. Lin (2001b) ejemplifica el generador de nombres de la siguiente forma:

Tabla 3

\section{Generador de posiciones de Lin (2001b)}

\begin{tabular}{|c|c|c|c|c|c|c|c|c|}
\hline \multicolumn{9}{|c|}{$\begin{array}{l}\text { De la siguiente lista de trabajos (mostrar la ficha de empleos), ¿podría decirme si conoce personalmente a al- } \\
\text { guien*? }\end{array}$} \\
\hline Trabajo & $\begin{array}{l}\text { 1. ¿Conoce } \\
\text { a alguien } \\
\text { que tenga } \\
\text { este empleo? }\end{array}$ & \begin{tabular}{|l|} 
2. ¿Cuánto \\
hace que \\
lolla conoce? \\
(núm. años)
\end{tabular} & $\begin{array}{l}\text { 3. ¿Cuál es } \\
\text { su relación } \\
\text { con esta } \\
\text { persona? }\end{array}$ & \begin{tabular}{|l|} 
4. ¿Cómo \\
de próximo \\
se siente \\
con esta \\
persona?
\end{tabular} & $\begin{array}{l}\text { 5. ¿El sexo } \\
\text { de esta } \\
\text { persona } \\
\text { es...? }\end{array}$ & $\begin{array}{l}\text { 6. ¿Trabaja } \\
\text { en ...? }\end{array}$ & \begin{tabular}{|l|} 
7. ¿Podría \\
encontrar a \\
una persona \\
asía a través \\
de algún \\
conocido \\
persona $M$ ?
\end{tabular} & $\begin{array}{l}\text { 8. Repetir } \\
\text { 2-6 para la } \\
\text { persona } M\end{array}$ \\
\hline \multicolumn{9}{|l|}{ A } \\
\hline \multicolumn{9}{|l|}{ B } \\
\hline \multicolumn{9}{|l|}{ C } \\
\hline$\ldots$ & & & & & & & & \\
\hline
\end{tabular}


Dado que el acceso a posiciones sociales superiores es un buen indicador de capital social, Lin ha desarrollado un indicador de upper accesibilitiy (acceso a posiciones superiores) para permitir la comparación.

No de extrañar que los estudios que utilizan esta propuesta hayan crecido rápidamente en los últimos años (Cf. Lin et. al. 2001). Flap y De Graaf (1986, citado en Van der Gaag y Snijders, 2004) también definieron de forma similar el capital social como el conjunto de recursos accesibles a través de relaciones determinadas, de forma que

$$
\mathrm{SC}=\sum_{i} \sum_{j} r_{i j} p_{i j}
$$

esto es, el capital social (SC) es igual al sumatorio de todos los alteri (i) que controlan o acceder un tipo de recurso $j$, siendo $r_{i j}$ la cuantificación de la posesión del recurso $j$ por $i$ y $p_{i j}$ la probabilidad que $i$ acceda al recurso $j$. De esta forma es necesario cuantificar por una parte los recursos de un tipo determinado poseídos por los alteri de una red personal y la probabilidad de ego de acceder a ese recurso.

Van der Gaag y Snijders (2004) proponen una forma alternativa de medir el capital social a la del generador de posiciones y es la del generador de recursos. El capital social medido de esta forma se expresa de la siguiente forma:

$$
\mathrm{SC}=\sum_{j} s_{j}
$$

Donde $j$ se refiere al recurso y $s_{j}$ indica una medida de la disponibilidad de ese recurso para una persona determinada. Al igual que en el caso del generador de posiciones, los autores proponen medir la intensidad de la relación para poder medir la capacidad de movilización de los recursos embebidos en la red social. Como ocurre con el generador de posiciones, es necesario disponer de una visión de conjunto de los recursos disponibles en una estructura local determinada para poder obtener indicadores fiables. A pesar de lo interesante de la propuesta, no ocultamos la dificultad existente a la hora de fundamentar el «mapa» de recursos accesibles. En todo caso, es de destacar que la encuesta estatal holandesa «Social relations and networks in the neighboorhood and the workplace: the Social Survey of the Networks of Dutch» (SNND) iniciada en 1999 incorpora generadores de nombres, generadores de posiciones y generadores de recursos (Flap et al., 1999). En la Tabla 4 se muestra un ejemplo de los generadores de recursos de esta encuesta.

Hasta aquí la revisión de las diferentes tradiciones de investigación. Veamos ahora qué conclusiones podemos extraer de las diferentes contribuciones realizadas. 
Tabla 4

Tabla de recursos. Adaptado de Flap et. al. 1999 (SNND)

\begin{tabular}{|c|c|c|c|c|c|c|}
\hline I. C & onoce a alguien que ... & No & Familiar & Amigo & Conoci & \\
\hline II .. & o podría ... & & & & & $\begin{array}{l}\text { ¿Ud. } \\
\text { mismo? }\end{array}$ \\
\hline 1 & ... reparar un coche, una bicicleta .. & $(0)$ & (1) & (2) & (3) & (4) \\
\hline 2 & ... tenga un coche. & $(0)$ & (1) & (2) & (3) & (4) \\
\hline 3 & ... sea hábil en reparaciones domésticas & $(0)$ & (1) & (2) & (3) & (4) \\
\hline 4 & $\begin{array}{l}\text {... pueda leer y escribir en una lengua } \\
\text { extranjera ... }\end{array}$ & $(0)$ & (1) & (2) & (3) & (4) \\
\hline 5 & ... pueda usar el ordenador ... & $(0)$ & (1) & (2) & (3) & (4) \\
\hline 6 & ... pueda tocar un instrumento ... & $(0)$ & $(1)$ & (2) & (3) & (4) \\
\hline 7 & ... conozca literatura ... & $(0)$ & (1) & $(2)$ & (3) & $(4)$ \\
\hline 8 & ... tenga el bachillerato ... & $(0)$ & (1) & (2) & (3) & (4) \\
\hline 9 & ... tenga estudios universitarios ... & $(0)$ & (1) & (2) & (3) & (4) \\
\hline 10 & ... lea un periódico de negocios ... & $(0)$ & (1) & (2) & (3) & (4) \\
\hline 11 & ... sea activo en una partido político ... & $(0)$ & (1) & (2) & (3) & (4) \\
\hline 12 & $\begin{array}{l}\ldots \text { tenga acciones por un valor mínimo } \\
\text { de } x \in\end{array}$ & $(0)$ & (1) & (2) & (3) & (4) \\
\hline 13 & ... trabaje en el ayuntamiento ... & (0) & (1) & (2) & (3) & (4) \\
\hline 14 & ... gane más de $y_{-} \ldots$ & (0) & (1) & (2) & (3) & (4) \\
\hline 15 & ... tenga una segunda residencia lejos ... & $(0)$ & (1) & (2) & (3) & (4) \\
\hline 16 & ... contrate gente a veces ... & $(0)$ & (1) & $(2)$ & (3) & (4) \\
\hline 17 & ... sepa mucho sobre las leyes del & (0) & (1) & (2) & (3) & (4) \\
\hline 18 & $\begin{array}{l}\text {... tenga buena relación con periódicos, } \\
\text { radio o emisoras de TV ... }\end{array}$ & $(0)$ & (1) & (2) & (3) & (4) \\
\hline 19 & ... sepa sobre fútbol ... & $(0)$ & (1) & $(2)$ & (3) & (4) \\
\hline 20 & ... sepa de temas fiscales ... & $(0)$ & (1) & (2) & (3) & (4) \\
\hline
\end{tabular}

\section{LAS CARACTERÍSTICAS DE LAS REDES PERSONALES}

En este apartado intentaremos sistematizar los principales hallazgos en nuestro campo siguiendo el esquema siguiente: tamaño de la red personal, composición de los atributos de los alteri, tipos de relaciones, contenido de las relaciones, estructura de la red personal y dinámica de las redes personales.

\section{Tamaño de la red personal}

Anteriormente hemos tenido oportunidad de revisar la literatura centrada en investigar esta cuestión. Como vimos, la respuesta a la pregunta ¿a cuántas per- 
sonas conoces? depende de cómo definamos «conocer», tanto más teniendo en cuenta que los dispositivos informáticos de los que disponemos (agendas electrónicas, programas de networking, etc.) nos permiten almacenar, comunicar e identificar «contactos» de forma casi instantánea. Utilizando la definición de contactos activos, definidos como personas con las que existe reconocimiento y accesibilidad mutuas y con las que se haya tenido alguna interacción en los dos últimos años, podemos decir lo siguiente:

1. La media de contactos activos es de aproximadamente 290 personas, con una desviación típica de 250.

Esta desviación está relacionada con la clase social y las profesiones o rangos ocupacionales asociados, de forma que las clase sociales altas y las personas con categorías ocupacionales superiores, sobre todo los representantes institucionales, disponen de redes más amplias (y con más recursos, lógicamente). Así,

1.1. La clase social y la categoría ocupacional influyen en el tamaño de las redes personales.

Dado que la mujer suele estar subrepresentada en el mundo del trabajo formal y de la política, no es de extrañar que en promedio las redes sociales de las mujeres sean más reducidas. nor.

1.2. La tamaño de las redes personales de las mujeres es, en promedio, me-

Igualmente sabemos que el tamaño de la red personal, incluso en su definición de contactos activos, va subiendo hasta la madurez, para decrecer a continuación. Por tanto,

1.3. El tamaño de la red personal está relacionado con la edad, con redes «máximas» en la madurez.

El registro sistemático que, cada vez más, tienen nuestras interacciones (correo electrónico, llamadas telefónicas, SMS, e incluso, conversaciones cara-acara o en grupo) permitirá avanzar mucho en el conocimiento del tamaño y dinámica de las redes personales en el futuro.

\section{Composición de la red personal}

Entendemos por composición de la red personal la distribución de los atributos de los alteri o relaciones de ego. La primera constante, con una larga tradición en Ciencias Sociales, es la homofilia (Blau, 1964), es decir, la tendencia a inte- 
ractuar con iguales. Lin (2001a) propone una interesante reformulación de esta idea fundamental en los siguientes términos: los iguales tienen en común el control de los recursos asociados con la posición social compartida por lo que las relaciones entre ellos son naturalmente expresivas, de socialización e instantáneas. Esta socialización natural $\mathrm{u}$ «homófila» tiene el objetivo de asegurar los recursos compartidos fortaleciendo los vínculos. En cambio la relación que se produce entre posiciones sociales desiguales es forzosamente intencional y «artificial», exige un costo y suele tener fines instrumentales. Esta relación es «heterófila». Los lazos fuertes y débiles de Granovetter (1973) pueden aproximadamente asimilarse a estos dos conceptos. En cualquier caso podemos afirmar que

2. Las relaciones tienden a ser homófilas, por lo que el sexo, la clase social, la profesión y otras características consideradas relevantes en un contexto dado (como el grupo étnico, la religión, el fenotipo) de una persona influirá en la composición de su red personal.

Así, las mujeres tenderán a tener más mujeres en su red personal, los compañeros de estudios tienden a permanecer más tiempo entre los contactos activos y seguramente se desarrollan pautas de consumo y gusto asociados a cada grupo que permiten el reconocimiento mutuo (Bourdieu, 1979). En el caso de los jóvenes, la probabilidad que se relacionen con otros de extracción social diferente y la probabilidad que cambie su estatus son más altas.

\section{Tipos de relaciones}

Aunque los tipos de relaciones se pueden multiplicar, normalmente se distingue en la literatura entre «familiares cercanos», «familiares», «mejores amigos», «buenos amigos», «compañeros de trabajo», «vecinos» y «conocidos». Naturalmente, los compañeros de trabajo, los amigos y los compañeros de ocio pueden ser los mismos. La literatura centrada en el apoyo social ha identificado (con los generadores de nombres utilizados) que los familiares en Estados Unidos y Canadá representan aproximadamente el $50 \%$ de la red personal. Sin embargo, como sabemos, esta literatura solamente estudia una pequeña submuestra de la red personal, por lo que el sesgo para que aparezcan solamente lazos fuertes es muy alto. En la misma línea, un reciente estudio realizado en Cataluña ( $C f$. Wellman et. al. 2002) arroja una proporción de familiares en la red menor del $50 \%$, aunque éstos persisten más en la red que los amigos, más sensibles al traslado de la residencia. Como veremos a continuación, esta importancia de los familiares en las redes personales en diferentes países no supone que tengan un rol activo en el apoyo social cotidiano, en el que otros tipos de relaciones pueden ser importantes (como vecinos y compañeros de trabajo).

Siguiendo el mismo método, en el caso de minorías étnicas (como los hispanos en California) el porcentaje de familiares puede llegar al $70 \%$. Este dato 
(Schweizer et. al, 1998) es interesante pues nos permite hipotetizar un rasgo de las redes personales de comunidades inmigradas ( $C f$. Maya Jariego 2002 para una tipología de las redes personales en España).

Sin embargo, estudios orientados a la estimación de la redes personales globales (Cf. McCarty et. al. 1997) sugieren que

3. La proporción de familiares se sitúa, en promedio, en torno al $25 \%$, la proporción de compañeros de trabajo alrededor $20 \%$ y la de vecinos en torno al $6 \%$.

Igualmente, estos estudios sitúan en más del $50 \%$ de las redes personales fuera de los lugares de residencia.

\section{Contenido de las relaciones}

En la literatura suele distinguirse entre apoyo social (cotidiano y de emergencia), de socialización o confidencia y relaciones instrumentales. Otra vez el género es importante:

\section{Las redes con más mujeres tienden a contener más apoyo social.}

Además, es interesante ver que el apoyo cotidiano tiende a ser provisto por vecinos y compañeros de trabajo más que por familiares, si bien como sabemos al final de la vida, con el aumento de la dependencia, los roles de apoyo se fortalecen. No obstante, el contenido de la relación con los hijos/as adultos es claramente de soporte. Así, podemos afirmar lo siguiente:

5. Los hijos/as (políticos o no) adultos tienden a proveer de apoyo social a los padres; esta relación será de más apoyo cuanto más densidad de relaciones haya con otros miembros de la red.

Por último,

6. El rol de los amigos tiende a ser de socialización y confidencia.

\section{Estructura de la red personal}

Sabemos que la diversidad en la red personal es un indicador de capital social, pues puede suponerse que diferentes grupos sociales controlen diferentes recursos, aunque lógicamente esta diversidad debería estar ponderada por el lugar ocupado en la jerarquía social. El estudio de la estructura de las redes personales justo acaba de comenzar en el sentido de disponer de información de las relaciones entre sí de una muestra representativa de los alteri de ego, y no solamente 
de los lazos fuertes. Nos ocuparemos de esta cuestión a continuación. Por ahora podemos afirmar que, efectivamente,

7. Las redes personales disponen de una fuerte estructura de centro-periferia, con un centro denso y una periferia más dispersa.

Además (McCarty et. al. 1997),

8. La densidad de las redes personales es en promedio del $30 \%$.

Seguramente las redes de las mujeres tienden a ser más densas por una variedad de razones (más frecuencia de contacto, más apoyo social, redes más pequeñas).

\section{Dinámica}

Las redes personales, incluso los amigos considerados «de toda la vida» cambian con el tiempo. Los cambios más drásticos se dan en la juventud y con el mundo del trabajo en la forma que ya sabemos: una punta de contactos en la madurez que decae con el tiempo. Los cambios del estatus matrimonial (matrimonio, viudez, separación) y los cambios de residencia afectan notablemente a los contactos (reduciéndolos). Sin embargo, estos cambios siguen en general la regla siguiente (Suitor et. al, 1997; Morgan et. al., 1997):

8. El centro de la red es muy estable a lo largo del tiempo y alrededor de $1 / 3$ de la periferia cambia con el tiempo.

Relacionada con la anterior:

9.1. Los lazos fuertes (más intensidad, frecuencia y más antigüedad de la relación) son más estables que los débiles.

Y lo que ya sabemos:

9.1. Los lazos familiares persisten más que el resto de lazos.

En este punto no podemos dejar de mencionar la interesante propuesta de $\mathrm{Fe}$ rrand et. al. (1999) según la cual a lo largo de la vida se producen los siguientes cambios en los roles:

10. Los roles dominantes ( amistad $=$ confidencia $;$ familia $=$ apoyo social) son sustituidos durante la madurez en parte (compañeros de trabajo = confidencia; vecinos = apoyo social) pero vuelven a recobrar fuerza a partir de los 60 . 


\section{PRINCIPALES SESGOS EN EL RECUERDO DE LAS RELACIONES PERSONALES}

La memoria es selectiva. En el caso de las relaciones sociales, la memoria está afectada por un conjunto de sesgos bien conocidos que exponemos a continuación.

La serie de estudios sobre la fiabilidad de los informes de los informantes sobre sus relaciones conocida como BKS (iniciada por Killworth y Bernard, 1976) puso de manifiesto la existencia de una discrepancia fundamental entre lo que la gente dice y lo que la gente hace.

Esta tendencia se corrige, no obstante, en las relaciones a largo plazo, de forma que los informantes tienden a reproducir en sus informes este tipo de relaciones (Freeman y Romney, 1987). De entre los múltiples sesgos hemos seleccionado aquéllos ampliamente contrastados y que son de utilidad a la hora de interpretar los informes de ego sobre sus redes personales.

1. Existe una tendencia generalizada a vernos más centrales de lo que realmente somos cuando informamos de nuestras relaciones y de las relaciones de los demás.

Ronald Burt (1994) llama a este efecto tertius gaudens, el tercero gana o disfruta, es decir ego al informar sobre otros alteri tiene tendencia a conectarlos a través suyo. Este efecto ( $C f$. Johnson, y Orbach, 2002) se complementa con otro bien conocido desde Festinger (1950) y Warner (1963),

2. Existe una tendencia generalizada a informar de las relaciones de estatus superior y olvidar las de estatus inferior.

A estos dos efectos generales, añadiremos un conjunto de factores que afectan a la fiabilidad de los informes sobre relaciones sociales:

3. Cuanta más interacción en la relación, más fiable será el informe de la relación social (Romney y Faust, 1983).

4. Las posiciones más centrales en una estructura social disponen de mejor información sobre la relaciones sociales efectivamente existentes.

Este hallazgo de Krackhardt (1990) es interesante, porque implica que las personas periféricas disponen de información bastante deficiente de lo que realmente pasa. De hecho, existe un sesgo que disimula nuestra común falta de información conocido como «rellenar los blancos» (fill-in-the-blanks, Freeman, 1992):

5. Las relaciones que no se conocen se suponen utilizando una característica en común, como trabajar en la misma empresa o haber estudiado en el mismo lugar. 
A estos efectos cabe añadir los sesgos que se producen por asociación, de forma que al recordar una persona, ésta nos lleva a la memoria otra con la que (de nuevo) se comparte una característica (Brewer 1994, 1997, 2000; Feld y Carter 2002).

En general podemos decir que los informantes tienen, desde su visión egocéntrica, una imagen cognitiva de la estructura social en la que se hayan insertos, y que sus informes tienden a reflejar esta imagen, la cual, como acabamos de decir, está fuertemente condicionada por la propia posición de ego en esa estructura.

\section{DE LA ESTIMACIÓN DEL TAMAÑO A LA ESTIMACIÓN DE LA ESTRUCTURA DE LAS REDES PERSONALES}

Recientemente. McCarty (2002b) ha sugerido un cambio en el enfoque del estudio de redes personales, pasando de la estimación del tamaño a la estimación de la estructura de las redes personales. La pregunta es: ¿cuál es el mínimo de número de alteri sobre los que hay que indagar para disponer de una idea aproximada de la estructura de la red personal? McCarty sugirió que un número entre 40 y 60 personas tendría que ser suficiente si el generador de nombres utilizado permite obtener una lista libre de personas de todas las categorías sociales. Por ejemplo:

Por favor, díganos una lista de 40 personas que Ud. conozca. Intente que todas las categorías importantes de personas (parientes, amigos, compañeros, vecinos...) estén representados en la lista.

El generador utilizado tiende a producir lazos íntimos al principio, pero la lista es lo suficientemente larga como para recoger información de diferentes áreas de la estructura de las redes personales, incluidos los lazos débiles. A continuación es necesario indagar sobre quién conoce a quién, aparte de recoger datos atributivos sobre ego y los alteri. Esta parte es la más larga pues una lista de 40 personas implica 780 preguntas de si dos personas se conocen o tienen algún tipo de relación que interese recoger. Para recoger esta información es necesaria la ayuda del programa Egonet. Por último, se presenta un informe a ego sobre su propia red personal. En todos los casos, los informantes se han mostrado sorprendidos por la calidad de la información mostrada sobre sus propias redes (Aguilar y Molina, 2004).

Una segunda alternativa (McCarty, 2004) para recoger información sobre la estructura social es, también con la ayuda de un programa informático, ir preguntando a ego por diferentes personas con las que tiene lazos fuertes, las relaciones entre éstas, e ir explorando a continuación otros círculos no conectados con los anteriores, de forma que se pueda recoger una estimación de la estructura de la red personal. Los resultados obtenidos por ambos métodos son similares, si bien en el segundo el cansancio de las personas encuestadas es mucho menor 
(gracias a una interfaz gráfica que permite ir construyendo al informante la visión de su propia red personal).

De esta forma, ya sea por el primer o segundo método, a diferencia de las encuestas reseñadas en los apartados anteriores, no solamente se recoge información atributiva sobre ego y sus alteri, sino que es posible obtener una imagen empírica de la red personal de ego. Las medidas estructurales de la red (densidad, componentes, cliques, centralidad, conectividad local, etc.) se añaden así a las medidas sobre la composición de las redes personales, permitiéndonos formular nuevas preguntas como por ejemplo la relación existente entre la diversidad de la red social (en términos atributivos y estructurales) con la identidad étnica.

Veamos un ejemplo.

En la ilustración siguiente (Ilustración 7) la imagen de la izquierda corresponde a una mujer (ego no aparece en el gráfico). La red es bastante densa (40\%) con una persona central que conecta el mundo del trabajo con el de la
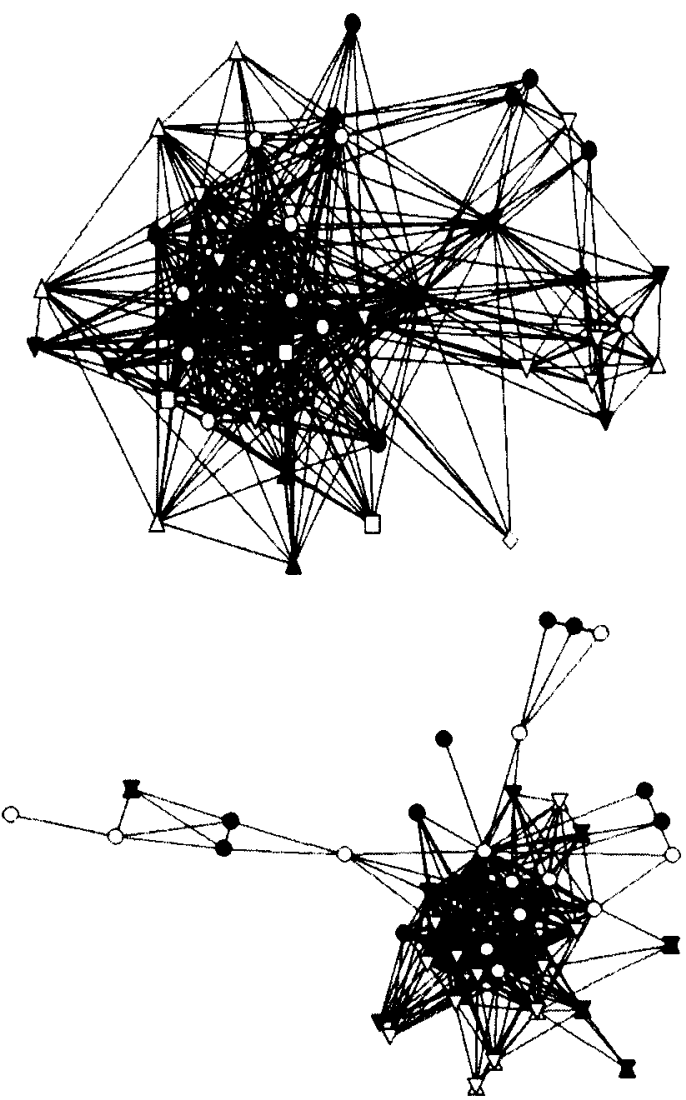

Ilustración 7. Dos redes personales de dos jóvenes de Sarajevo. Los símbolos indican diferentes grupos étnicos (serbios, croatas, bosnios, etc.) 
familia y amigos, que de hecho es su marido (ambos trabajan juntos). El núcleo intimo esta formado por bosnios ( $42 \%$ ) y bosníacos (bosnios musulmanes) $(33 \%)$, mientras que en la periferia hay más variedad (15\% de croatas, $6 \%$ de serbios).

La imagen de la derecha corresponde a un varón. Su red presenta menor densidad $(20 \%)$ y tiene también un núcleo denso formado por bosníacos $(22 \%$, sus familiares directos), bosnios ( $56 \%$ ) y otros (22\%) que, en su caso, son extranjeros. Los dos grupos de personas que observamos separados del centro están constituidos por relaciones de trabajo y de estudios.

Estas redes diferentes nos pueden llevar a formular nuevas preguntas como, por ejemplo, si hay alguna relación entre características de la red personal y discursos étnicos. Todo parece indicar que es así.

Como puede suponerse fácilmente, El método sugerido para analizar la estructura de redes personales tiene un amplio rango de aplicaciones. Los gestores de organizaciones son receptivos a esta evaluación de sus redes personales porque les permite disponer de una aproximación a su capital social y los círculos sociales en los que se mueven. También, los trabajadores sociales pueden usar esta herramienta para diagnosticar los recursos sociales de las personas que atienden, intentando mejorar su situación, y así sucesivamente.

Pensamos sinceramente que la acumulación de este tipo de información de redes personales en contextos culturales diversos puede complementar los estudios clásicos sobre redes personales y permitir investigar, no solamente la relación entre estructura, personalidad y conducta, sino también aproximarnos a las estructuras meso en las que estas redes se hayan inmersas. Veamos esta cuestión a continuación.

\section{UN OBJETIVO TEÓRICO: ESTRUCTURAS «MESO»}

En este apartado apuntamos solamente una línea de trabajo para identificar estructuras «meso» a partir de la combinación de análisis egocéntricos y sociocéntricos. Recordemos la imagen de un modelo hipotético de una estructura local que recogía la propuesta de Nan Lin (Ilustración 8).

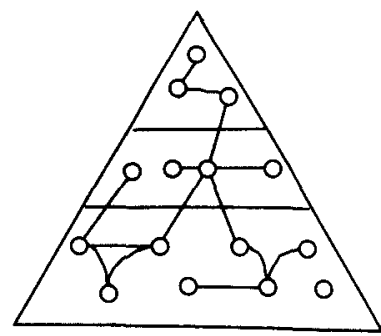

Ilustración 8. Estructura local. 
Como puede verse, existen relaciones entre personas de los mismos estratos y, aunque de forma más limitada, relaciones entre personas de diferentes estratos. Sabemos que a partir de una muestra de la estructura local podemos investigar las redes personales de diferentes estratos al estilo de los Estudios de Comunidad o de Capital social clásicos. A continuación, podemos identificar las personas más nominadas (indegree o grado nodal de entrada) y estudiar la estructura de su redes personales de la forma descrita en el apartado anterior. Costenbader y Valente (2003) han mostrado de forma convincente que esta medida es un indicador muy robusto que permite aproximarse a los elementos más centrales de una red social determinada en contexto de severa falta de información ( $C f$. también Feld et. al. 2002).

Agregando a continuación las diferentes redes personales de las personas más centrales en una única red social a partir de las personas nominadas por más de un informante, dispondremos de una muestra de la red social de la estructura local. Es decir, agregando las redes personales en una sociomatriz que relacione a las personas en común (y conservando las variables atributivas de los alteri, entre ellas la posición ocupada en la jerarquía local) podremos construir una matriz $M$ de la estructura local. Si el informante $i$ indica la existencia de una relación entre $j$ y $k$ en su red personal diremos que $(j, k)=1$ y que $j=1$ y $k=1$. Entonces la sociomatriz $M$ estará constituida por

$$
M=\sum_{i j k} \text { si } j>n \text { o } k>n \text {, siendo } n>1
$$

$n$ es el umbral introducido por el investigador.

En el caso que diferentes informantes ofrezcan informes contradictorios sobre $(j, k)$, conservaremos el informe del informante con el lazo más fuerte o la interacción más alta con $j$ y/o $k$. La existencia de informes contradictorios entre egos con lazos fuertes con $j$ y/o $k$ son, en la práctica, poco frecuentes.

Ahora bien, la visión de la estructura local de la Ilustración 8 es incompleta.

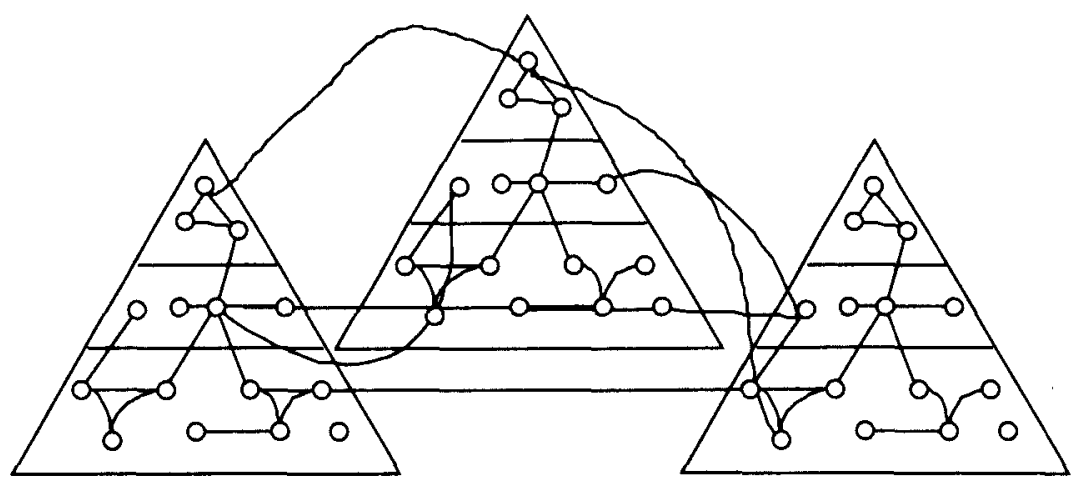

Ilustración 9. Jerarquias locales dentro y fuera de un campo político. 
En la Ilustración 9 podemos apreciar cómo las jerarquías locales tienen conexiones entre ellas, dentro y fuera de un campo político definido (un Estado o una asociación de Estados, por ejemplo). Estas jerarquías locales pueden estar a su vez jerarquizadas o no entre ellas, en función del nivel de centralización de la estructura política. Esta realidad se transforma naturalmente a lo largo de la dimensión temporal. Así, la matriz $M$ puede dar cuenta de las relaciones localescosmopolitas que tienen lugar en la estructura local analizada (Ferrand, 2002; Merton, 1968), pues las relaciones recogidas de las redes personales no se limitan a la estructura local.

Pero esto no es suficiente. Los individuos pertenecen simultáneamente a redes y a grupos, como, por ejemplo, organizaciones locales. Por ello, siguiendo la propuesta de Breiger (1974) la matriz $M$ debe complementarse con una matriz $G$ de organizaciones locales (es decir, una matriz de organizaciones $\mathrm{x}$ organizaciones) de forma que sea posible construir una matriz $A$ de afiliaciones de individuos a grupos u organizaciones (personas $\mathrm{x}$ organizaciones). Es decir, indicar la pertenencia de los individuos con grado nodal de entrada o indegree $>n$ a la red de organizaciones locales. Los niveles micro y meso estarían pues integrados. Este tipo de datos es susceptible de ser analizado por Galois lattices (Mohr, 2000; Breiger \& Mohr, 2004), técnica que permite poner de manifiesto las abstracciones subyacentes en una tabla de datos a través de una red de conceptos.

A la hora de evaluar el impacto de esta sucesiva integración de los niveles (las interacciones o las relaciones, los individuos, las redes, las redes de organizaciones) será necesario utilizar los modelos estadísticos multinivel (Van Duijn, et. al., 1999; Wellman y Frank, 2001), los cuales asumen que los datos no son independientes sino que están anidados de forma jerárquica, por lo que es posible identificar el efecto de cada uno de los niveles en las variables dependientes consideradas. Estos modelos estadísticos son más adecuados para el estudio de las redes sociales, por la interdependencia que implican los datos reticulares. El análisis de estructuras meso a través de las redes personales está, pues, a nuestro alcance. 


\section{BIBLIOGRAFÍA}

Aguilar, C. y Molina, J. L. (2004): «Ethnic identities and personal networks among young people in Sarajevo», ponencia presentada en la XXIV International Sunbelt Social Network Conference, Portoroz, Slovenia, Mayo, pp. 12-16.

BARABÁSI, A.-L. (2002): Linked. The New Science of Networks. Cambridge, MA, Perseus Publishing.

BARNES, J. (1954): «Class and committees in a Norwegian Islan Parish», Human Relations, vol. 7, n. 1, pp. 39-58).

Bernard, R. H.; Killworth, P. D.; Evans, M. J.; McCarty, C. and Shelley, G. A. (1988): «Studying social relations cross-culturally» Ethnology, vol. 27, 2, April, pp. 155-179.

Bernard, R. H.; Johnsen, E. C.; Killworth, P. D.; McCarty, C.; Shelley, G. A. and RoBINSON, S. (1990): «Comparing four different methods for measuring personal social networks", Social Networks 12, pp. 179-215).

Bernard, R. H.; Killworth, P. D.; Johnsen, E. C.; Shelley, G. A. and McCarty, C. (2001): «Estimating the Ripple Effect of a Disaster», Connections 24(2), pp. 18-22.

BlaU, P. M. (1964): Exchange and Power in Social Life. New York, John Wiley.

BoISSEVAIN, J. and ClYDE, M. J. (1973): Network Analysis Studies in Human Interaction. London, Mouton \& Co.

BoTt, E. (1955): «Urban Families: Conjugal Roles and Social Networks», Human Relations, vol VIII, pp. 345-384.

- (1957): Family and Social Network. London, Tavistock Institute of Human Relations.

BouRdieu, P. (1977): Outline of a Theory of Practice. New York, Cambridge University Press.

- (1988): La distinción: criterio y bases sociales del gusto [La Distinction, 1979]. Madrid, Taurus.

BREIGER, R. y MOHR, J. W. (2004): «La dualidad y la agregación de categorías sociales», Revista Redes, Volumen 5 \#4, Enero-Febrero. http://revista-redes.rediris.es

BREWER, D. D. y YANG, B. L. (1994): «Patterns in the recall of persons in a religious community», Social Networks 16, pp. 347-379.

BREWER, D. D. (1997): «No associative biases in the first name cued recall procedure for eliciting personal networks», Social Networks 19, pp. 345-353.

- (2000). «Forgetting in the recall-based elicitation of personal and social networks», Social Networks 22 , pp. 29-44.

BuRT, R. S. (1992): Structural Holes: The Social Structure of Competition. Cambridge, Harvard University Press.

BURT, R. S. and RoNCHI, D. (1994): «Measuring a large network quickly», Social Networks, vol. 16, No. 2, April.

CASTELlS, M. (1996): The Information Age: Economy, Society and Culture. The Rise of the Network Society, Vol. I. Cambridge, MA; Oxford, UK, Blackwell.

COLEMAN, J. (1988): «Social capital in the creation of human capital», American Journal of Sociology, 94.

- (1990): Foundations of Social Theory. Cambridge, MA, Harvard University Press.

COSTENBADER, E. and VAlENTE, T. W. (2003): «The stability of centrality measures when networks are sampled», Social Networks, 25(4), pp. 283-307.

DE FEDERICO, A. (2002): «Presentacion: Tendiendo puentes, de Lilnet a Redes», Revista Redes, vol. 3, \#1, Sept-Nov. http://revista-redes.rediris.es 
- (2004): «Los espacios sociales de la transnacionalidad. Una tipología de la integración relacional de los migrantes», ponencia presentada en la XXIV International Sunbelt Social Network Conference, Portorož, Slovenia,12-14 de Mayo.

EPSTEIN, A. L. (1957): «Urban Communities in Africa» en Gluckman, Max, ed. (1964), Closed Systems and Open Minds: The Limits of Naïvety in Social Anthropology. London, Oliver \& Boyd.

- (1961): «The network and urban social organization», Rhodes-Livingstone Journal, 29, pp. 29-62.

- (1969): «Gossip, Norms and Social Networks» en Mitchell, Clyde J., Social Networks in Urban Situations. Analyses of Personal Relationships in Central African Towns. Manchester, Manchester University Press.

Fisher, C. (1982): To Dwell Among Friends.Personal Networks in Town and City. Chicago, University of Chicago Press.

FELD, S. L. and CARTER, W. C. (2002): «Detecting measurement bias in respondent reports of personal networks», Social Networks 24, pp. 365-383.

Ferrand, A.; Mounier, L. y Degenne, A. (1999): «The Diversity of Personal Networks in France: Social Stratification and Relational Structures» en Wellman, Barry, Networks in the Global Village. Life in Contemporary Communities. Oxford, Westview Press.

FERRAND, A. (2002): «Las comunidades locales como estructuras meso», Revista Redes, Vol. 3, \#4, Septiembre-Noviembre. http://revista-redes.rediris.es

FESTINGER, L.; SCHACHTER, S. and BACK, K. (1950): Social Pressures in Informal groups: A Study of Human Factors in Housings. Stanford, Stanford University Press.

FELD, S. L. and CARTER, W. C. (2002): «Detecting measurement bias in respondent reports of personal networksm. Social Networks 24, pp 365-383.

FLAP, H. D. y DE GRAAF, N. D. (1986): «Social capital and attained occupational status», Netherlands Journal of Sociology 22, pp. 145-161.

FLAP, H.; SNIJDERS, T.; VólKER, B. y VAN DER GAAG, M. (1999): «Measurement instruments for social capital of individuals», SSND.

Freeman, L. C.; RomNEY, A. K. and Freeman, S. C. (1987): «Cognitive structure and informant accuracy», American Anthropologist, 89, pp. 310-325.

FREEMAN, L. C. and THOMPSON, C. R. (1989); «Estimating acquaintanceship volume» en Kochen, M. (ed.), The Small World. Norwood, NJ: Ablex, pp. 147-158.

FREEMAN, L. C. (1992): «Filling in the blanks: a theory of cognitive categories and the structure of social affilitation», Social Psychology Quarterly, 55 (2), pp. 118-127.

- (2004): The Development of Social Network Analysis. A Study in the Sociology of Science. Vancouver, Empirical Press.

Fu, Yang-chih (2005): «Measuring personal networks with daily contacts: a single-item survey question and the contact diary", Social Networks, Volume 27, pp.169-186.

GRANOVETTER, M. (1973): «The Strength of Weak Ties», American Journal of Sociology $78(6)$, pp. 1360-1380.

GROSSETTI, M. (2004): «Are Frenchs Networks Different?», ponencia presentada en la XXIV International Sunbelt Social Network Conference, Portorož, Slovenia. 12-16 de Mayo.

GUREVICH, M. (1961): The social structure of acquaintanceship networks. MIT: Tesis doctoral.

JoHNSON, J. C. and ORBACH, M. K. (2002): «Perceiving the Political Landscape: Ego Biases in Cognitive Political Networks», Social Networks 24, pp. 291-310. 
KAPFERER, B. (1972): Strategy and transaction in an African factory: African workers and Indian management in a Zambian town. Manchester, Manchester University Press.

- (1973): «Social network and conjugal role in urban Zambia: towards a reformulation of the Bott hypothesis» en Jeremy Boissevain \& J. Clyde Mitchell, Network Analysis Studies in Human Interaction, Mouton \& Co.

KILLWORTH, P. D. y BERNARD, H. R. (1976): «Informant accuracy in social network data», Human Organization, 35, pp. 269-286.

- (1978): «The Reverse Small World Experiment», Social Networks, vol. 1, pp. 159-192.

KillworTh, P. D.; Bernard, H. R., y MCCARTY, C. (1984): «Measuring Patterns of Acquaintanceship», Current Anthropology, vol. 23, pp. 318-397.

Killworth, P. D.; Johnsen, E. C.; McCarty, C.; Shelley, G. A. y Bernard, H. R. (1998): «A social network approach to estimating seroprevalence in the United States», Social Networks 20, pp. 23-50.

Killworth, P. D.; Johnsen, E. C.; Bernard, H. R.; Shelley, G. A. y McCarTy, C. (1990): «Estimating the size of personal networks», Social Networks, vol. 12, pp. 289-312.

KRACKHARDT, D. (1990): «Assesing the Political Landscape: Structure, Cognition, and Power in Organizations», Administrative Science Quarterly, No. 35, pp. 342-69.

Laumann, E. O. (1973): Bonds of Pluralism: The Form and Substance of Urban Social Networks. New York, John Wiley.

LaumanN, E. O.; Marsden, P. V. and Prensky, D. (1983): «The Boundary Specification Problem in Network Analysis» in Burt, Ronald S. and Michael J. Minor, (eds.), Applied Network Analysis. Beverly Hills, Sage. pp. 19-34.

LiN, N. (1982): «Social Resources and Instrumental Action» en Marsden, Peter V. y Lin, Nan (eds.), Social Structure and Network Analysis. London, Sage Publications.

- (2001a): Social Capital: A Theory of Social Structure and Action. Cambridge, Cambridge University Press.

- (2001b): «Building a Network Theory of Social Capital», en Lin, Nan, Cook, Karen \& Burt, Ronald S. (eds.). Social Capital. Theory and Research. New York, Aldine de Gruyter.

LIN, N.; COOK, K, and BURT, R. S. (2001): Social Capital. Theory and Research. New York: Aldine de Gruyter.

LITwIN, H. (1996): The Social Networks of Older people: A Cross-National Analysis. London, Praeger.

LoNKILA, M. (1997): «Informal Exchange Relations in Post-Soviet Russia: A Comparative Perspective», Sociological Research Online, vol. 2, no. 2.

- (1998): «Continuity and change in social networks of St. Petersburg Teachers, 19931996», Connections 21(1):62-86. INSNA.

LOZARES, C. (2003): «Valores, campos y capitales sociales», Revista Redes, vol. 4, \#2, Jun. http://revista-redes.rediris.es

MAYA JARIEGO, I. (2002): «Tipos de redes personales de los inmigrantes y adaptación psicológica», Revista Redes, vol. 1, \#4, enero http://revista-redes.rediris.es

MAYA JARIEGo, I. and PeTrIZZO PÁEZ, M. A.: El software social y la capitalización de las redes personales.

Maya Jariego, I.; Martínez Garcia, M. F. y Garcia Ramirez, M. (1999): «Cadenas migratorias y redes de apoyo social de las mujeres peruanas en Sevilla», Demófilo. Revista de cultura tradicional de Andalucía, 29 (87-105). 
McCarty, C.; Bernard, H. R.; Killworth, P. D.; Shelley, G. A. y Johnsen, E. C. (1997): «Eliciting representative samples of personal networks», Social Networks, 19, pp. 303-323.

McCarty, C.; Killworth, P. D.; Bernard, R. H.: Johnsen, E. C. y Shelley, G. A. (2000): «Comparing Two Methods for Estimating Network Size», Human Organization, 60, pp. 28-39.

McCARTY, C. (2002a): «Social Network Analysis and Community Studies», Encyclopedia of Community (en prensa).

- (2002b). «Structure in Personal Networks», JoSS. <http://www.library.cmu.edu:7850/ JoSS/McCarty/McCarty.htm>

- (2004). «Visualization of Personal Networks», ponencia presentada ene el XXIV International Sunbelt Social Network Conference, Portorož, Slovenia, May 12-16.

MERTON, R. K. (1968): «Patterns of Influence: Local and Cosmopolitan Influentials», en: Social Theory and Social Structure. New York, The Free Press.

Milgram, S. (1967): «The small world problem», Psychology Today, 1, pp. 61-67.

MORENO, J. (1976): Fundamentos de sociometría [Who shall survive? Foundations of Sociometry, 1934]. Buenos Aires, Paidós.

MrTCHELl, J. C. (1969): «The Concept and Use of Social Networks», in: J. Clyde Mitchell (ed.), Social Networks in Urban Situations: Analyses of Personal Relationships in Central Towns. Manchester, Manchester University Press.

MoHR, J. W. (2000): «Structures, institutions, and cultural analysis», Poetics, Volume 27, Issue: 2-3, March, pp. 57-68.

MolinA, J. L. y ALAYO I GIL, A. (2001): «Reciprocity today: the network of domestics units and public services in two collectives in Vic (Barcelona)». European Science Foundation - SCSS Exploratory Workshop «Reciprocity as a Human Resource». Barcelona, pp. 12-15, Septiembre.

Molina, J. L. y AGuilar, C. (2004): «Redes sociales y antropología: un estudio de caso (redes personales y discursos étnicos entre jóvenes en Sarajevo)». Barcelona, Editorial Icària (en prensa).

Morgan, D. L., Neal, M. B. and Carder, P.: «The stability of core and peripheral networks over time», Social Networks, 19, pp. 9-25.

NADEL, S. F. (1957): Theory of social Structure. London, Cohen and West.

NAROTZKY, S. y MORENO, P. (2002): «Reciprocity's dark side. Negative reciprocity, morality and social reproduction», Anthropological Theory, 2(3), pp. 281-305.

Newman, M. E. J. (2001): «Ego-Centered Networks and the Ripple Effect.» <http://www.santafe.edu/sfi/publications/Abstracts/01-11-066abs.html> [también publicado en Social Networks 25, 1, pp. 83-96, 2003).

- (2003): "The structure and function of networks», SIAM Review, 45, pp. 167-256. http://arxiv.org/abs/cond-mat/0303516/

Petrizzo Páez, M. y Maya Jariego, I. (2004): «La red de Matrix. ¿En los límites de lo posible?», Revista TEXTOS de la CiberSociedad, 4. Descifrando MATRIX. Número monografico http://www.cibersociedad.net

Pool, I. S. and KochEN, M. (1978): «Contacts and influence», Social Networks, 1, pp. 551.

RADCLIFFe-Brown, A. R. (1940): «On Social Structure», Journal of the Royal Anthropological Society of Great Britain and Ireland, 70, pp. 1-12.

REDFIELD, R. (1960): The Little Community and Peasant Society and Culture. Chicago, Phoenix Books. 
ReQuena SAntos, F. (1996): «Redes sociales y cuestionarios», Cuadernos Metodológicos, 18. Madrid, CIS.

- (2003): Análisis de Redes Sociales: Orígenes, teorías y aplicaciones. Madrid, Centro de Investigaciones Sociológicas.

RodRigueZ, J. A. (1995): «Análisis estructural y de redes». Cuadernos Metodológicos, 16. Madrid, CIS.

RomNEY, A. K. and FAUST, K. (1983). «Predicting the structure of a communication network from recalled data», Social Networks, 4, pp. 285-304.

ScotT, J. (1991): Social Network Analysis. A Handbook. London, Sage Publications.

Shelley, G. A.; Bernard, H. R.; KILlWORTH, P.; Johnsen, E. and MCCARTY, C. (1995): "Who knows your HIV status? What HIV+ patients and their networks know about each other», Social Networks, 17, pp. 189-217.

SiMMEL, G. (1955): Conflict and the Web of Group-Affiliations. Glencoe, The Free Press.

SNIJDERS, T. A. B. (1992): «Estimation on the basis of snowball samples: how to weight?», Butlletin de Méthodologie Sociologique, 36, September, pp, 59-70.

SUITOR, J. J.: WELLMAN, B. and MORGAN, D. L. (1997): «It's about time: how, why, and when netwoks change», Social Networks, 19, pp. 1-7.

SCHweIzer, T.; SCHNEGG, M. and BERZBORN, S. (1998): «Personal Networks and social support in a multiethnic community of southern California», Social Networks, Volume 20, Issue 1, pp. 1-21.

TILBURG, T. G. van (1992): «Support networks before and after retirement», Journal of Social and Personal Relationships, 9, pp. 433-445.

- (1998): «Losing and gaining in old age: Changes in personal network size and social support in a four-year longitudinal study», Journal of Gerontology, 53B, pp. 313-323.

TönNIES, F. (1984): Comunitat i associació [Gemeinschaft und Gesellschaft, 1887]. Barcelona, Edicions 62-Diputació de Barcelona.

VAN DER GAAG, M. P. J. and SNIJDERS, T. A. B. (2004): «Proposals for the measurement of individual social capital»en Flap, H. D. and Volker, B. (eds.), Creation and returns of Social Capital. London, Routledge.

Van Duijn, M. A. J.: van Busschbach, J. T. and Snidders, T. A. B. (1999): Multilevel analysis of personal networks as dependent variables. Social Networks, 21(2), pp. 187-209.

WASSERMAN, St. and GALASKIEWICZ, J. (eds.) (1994): Advances in Social Network Analysis. Thousand Oaks, CA: Sage.

WARNER, W. L. (1963): Yankee City. New Haven \& London, Yale University Press.

WATTS, D. J. (1999): Small Worlds. The Dynamics of Networks between Order and Randomness. Princenton, Princenton University Press.

Wellman, B.; Carrington, P. J. and Hall A. (1988): «Networks as personal communities», en: Wellman \& Berkowitz (eds.), Social Structures; A Network Approach. Cambridge University Press, Cambridge, 1988, pp. 130-184.

WELLMAN, B. (1996): «Are personal communities local? A Dumptarian reconsideration», Social Networks, 18, pp. 347-354.

Wellman, B.; Wong, R. Y.; Tindall, D. and NAZER, N. (1997): «A decade of network change: turnover, persistence and stability in personal communities", Social Networks, 19, pp. 27-50.

Wellman, B. and Gulia, M. (1999): «Net-Surfers Don't Ride Alone: Virtual Communities as Communities», in: Wellman, Barry, ed., Networks in the Global Village. Life in Contemporary Communities. Oxford, Westview Press. 
Wellman, B. (2001): «Physical Place and CyberPlace: The Rise of Personalized Networking», en: Blokland, Talja \& Mike Savage, eds., «Networks, Class and Place», número especial del International Journal of Urban and Regional Research, 25(2), June, pp. 227-252.

Wellman, B.; Quan-HaAse, A.; Boase, J. and Wenhong, Ch. (2002): «Examining the Internet in Everyday Life», Keynote address to the Euricom Conference on e-Democracy, Nijmegen, Netherlands, October.

WHITE, H. C.; BOORMAN, S. A. and BREIGER, R. L. (1976): «Social Structure from Multiple Networks, I: Blockmodels of Roles and Positions», American Journal of Sociology, 81, pp. 730-780 


\title{
RESUMEN
}

En este trabajo se pretende ofrecer un estado de la cuestión del campo de estudio de las redes personales. En la primera parte se realiza una revisión de la literatura existente, dividida en cuatro líneas o tradiciones: la Escuela de Manchester, los Estudios de Comunidad, la Estimación de redes personales y el Capital Social. En la segunda parte se intenta mostrar de forma sistemática el conjunto de propuestas realizadas hasta el momento sobre las características, estructura, composición y dinámica de las redes personales. En la tercera parte se recogen los diferentes sesgos que se producen en el recuerdo de personas y relaciones sociales, los cuales hay que tener en cuenta a la hora de valorar los resultados de los diferentes estudios. Por último, en este trabajo se pretende contribuir, desde el estudio de las redes personales, a una línea de investigación orientada a estudiar fenómenos sociales de rango «intermedio» 0 «meso» es decir, fenómenos en los que se presentan simultáneamente interacciones individuales, instituciones y estructuras sociales observables empíricamente.

\section{PALABRAS ClAVE}

Redes personales, egoredes, análisis de redes sociales.

\begin{abstract}
The aim of this paper is to provide a state-of-the-art about the personal networks scientific field. The paper is divided in four parts. In the first part the literature is reviewed and classified in four traditions or Schools: the Manchester School, the Community Studies, the Personal Network estimation and the Social Capital. In the second part, the main contributions of those studies are summarized in a systematic way. The third part is devoted to the study of the bias involved in recalling personal relationships. Finally, we try to contribute to the study of meso structures or social phenomena where individual interactions, institutions and social structures are empirically observed.
\end{abstract}

\section{KEY WORDS}

Personal networks, egonetworks, social network analysis. 